ARTICLE

https://doi.org/10.1038/s41467-019-13962-0

\title{
FAM222A encodes a protein which accumulates in plaques in Alzheimer's disease
}

Tingxiang Yan ${ }^{1,80}$, Jingjing Liang ${ }^{2,80 *}$, Ju Gao (10 ${ }^{1,80}$, Luwen Wang ${ }^{1,80}$, Hisashi Fujioka ${ }^{3}$, The Alzheimer Disease Neuroimaging Initiative, Xiaofeng Zhu ${ }^{2 \star} \&$ Xinglong Wang ${ }^{1 \star}$

Alzheimer's disease (AD) is characterized by amyloid plaques and progressive cerebral atrophy. Here, we report FAM222A as a putative brain atrophy susceptibility gene. Our crossphenotype association analysis of imaging genetics indicates a potential link between FAM222A and AD-related regional brain atrophy. The protein encoded by FAM222A is predominantly expressed in the CNS and is increased in brains of patients with $A D$ and in an $A D$ mouse model. It accumulates within amyloid deposits, physically interacts with amyloid- $\beta$ $(A \beta)$ via its $N$-terminal $A \beta$ binding domain, and facilitates $A \beta$ aggregation. Intracerebroventricular infusion or forced expression of this protein exacerbates neuroinflammation and cognitive dysfunction in an AD mouse model whereas ablation of this protein suppresses the formation of amyloid deposits, neuroinflammation and cognitive deficits in the AD mouse model. Our data support the pathological relevance of protein encoded by FAM222A in AD.

\footnotetext{
${ }^{1}$ Department of Pathology, Case Western Reserve University, Cleveland, OH, USA. ${ }^{2}$ Department of Population and Quantitative Health Sciences, Case Western Reserve University, Cleveland, OH, USA. ${ }^{3}$ Electron Microscopy Core Facility, Case Western Reserve University, Cleveland, OH, USA. ${ }^{80}$ These authors contributed equally: Tingxiang Yan, Jingjing Liang, Ju Gao, Luwen Wang. A full list of consortium members appears at the end of the paper.

*email: jingjing.liang@case.edu; xxz10@case.edu; xinglong.wang@case.edu
} 
1 lzheimer's disease $(\mathrm{AD})$, the leading cause of dementia named for Dr. Alois Alzheimer, is characterized by pathologic hallmarks amyloid plaques and neurofibrillary tangles, and accompanied by other prominent pathological changes such as progressive atrophy of the brain, neuropil threads, dystrophic neurites, granulovacuolar degeneration, Hirano bodies, and cerebrovascular amyloid ${ }^{1}$. Amyloid plaques are spherical extracellular lesions composed of amyloid- $\beta$ (A $\beta)$ peptides, whereas neurofibrillary tangles are intracellular lesions made up of hyperphosphorylated form of the microtubuleassociated protein tau. Although many risk factors such as aging, lifestyle, and environmental factors are usually considered for the pathogenesis, $\mathrm{AD}$ is increasingly proposed to be a genetically dichotomous disease in the early-onset familial form showing classical Mendelian inheritance with little influence from the environment (EOAD), or in the late-onset sporadic form inherited in a non-Mendelian fashion (LOAD) ${ }^{2}$.

Less than $10 \%$ of $\mathrm{AD}$ cases are $\mathrm{EOAD}$ with only a small fraction caused by autosomal dominantly inherited genetic changes in amyloid precursor protein (APP), presenilin 1 (PS1) or presenilin 2 (PS2), all of which are responsible for the overproduction of $A \beta$ and the earlier formation of amyloid plaques ${ }^{3}$. Though more than $90 \%$ of $\mathrm{AD}$ cases are LOAD referred to as sporadic $\mathrm{AD}$ without family history, they have the similar clinical and pathologic phenotypes as EOAD and are heritable ${ }^{4}$. In the past decade, intensive efforts have been made to identify over 25 genes associated with $\mathrm{AD}^{5}$. In support of the dominant amyloid cascade hypothesis suggesting $A \beta$ deposition in the brain as the primary cause, a number of $\mathrm{AD}$-associated genes are enriched in the APP processing pathway, and involved in A $\beta$ overproduction and amyloid plaque deposition though their encoded proteins are usually not directly associated with amyloid plaques.

Quantitative structural magnetic resonance imaging (MRI) has been extensively used for assessment of AD-related structural differences in selective brain regions ${ }^{6}$. Genome-wide association studies (GWAS) using MRI measures have identified several AD risk variants ${ }^{7,8}$. Likewise, the MRI changes in statistically-defined regions of interest (ROI) were found closely associated with reported $\mathrm{AD}$ risk variants ${ }^{9,10}$. Genetic loci harboring variants can be associated with multiple, sometimes seemingly distinct, traits ${ }^{11,12}$. The test for such associations, i.e., cross-phenotype association test, has been increasingly employed to investigate the genetic overlap between multiple traits and diseases. Our previous study has developed a cross-phenotype association analysis (CPASSOC) that can integrate association evidence from GWAS summary statistics of multiple traits, either correlated, independent, continuous, or binary traits, and has been successfully used to identify four loci associated with hypertensionrelated traits missed by a single-trait analysis ${ }^{13}$. In this study, we performed CPASSOC analysis of MRI measures and genetic datasets, and identified a possible link between FAM222A and AD-related regional brain atrophy. To understand its pathological role in $\mathrm{AD}$, we investigated the protein encoded by FAM222A in patients with $\mathrm{AD}$ or transgenic mice for $\mathrm{AD}$, and found its characteristic accumulation within the center of amyloid deposits. Further mechanistic study revealed that this protein could physically interact with $A \beta$ and regulate $A \beta$ aggregation and amyloid formation. Our results therefore identify a protein that likely plays an important role in amyloidosis, a finding providing perspective for $\mathrm{AD}$ pathogenesis.

\section{Results}

Susceptibility of regional brain atrophy to FAM222A in AD. To identify brain atrophy-related imaging quantitative trait loci, we employed a genome-wide whole brain approach to analyze the imaging genetic dataset from the Alzheimer's Disease Neuroimaging Initiative (ADNI) (Supplementary Fig. 1a). After GWAS and the estimation of shared genetic contributions among 145 ROIs spanning the entire brain by linkage disequilibrium (LD) regression method ${ }^{14}$ (Supplementary Fig. 1b, c), we attempted to extract disease-related ROIs and detect genetic variants associated with them. With hierarchical clustering analysis on a genetic correlation network (Supplementary Fig. 1d-k), 16 modules of ROIs with high within-module genetic correlation were generated (Supplementary Fig. 1l, m). We further combined GWAS summary statistics of ROIs in each module using CPASSOC we developed $^{13}$. Previously reported AD top markers, APOE single nucleotide polymorphism (SNP) rs429358 ${ }^{15}$, TOMM40 SNP rs207565015, APOC1 SNP rs12721051 ${ }^{16}$, and rs117028417 on FAM222A were found in one module (Fig. 1, Supplementary Figs. 2, 3 and Supplementary Table 1), which consists of five ROIs including left hippocampus, right hippocampus, basal forebrain, entorhinal area, and planum polare, brain areas we know are affected by $\mathrm{AD}^{17-19}$ and well predict $\mathrm{AD}$ (Supplementary Fig. 4). SNP rs117028417 had a minor allele A (frequency $=0.044)$ with positive effects for all 5 ROIs in the ADNI cohort $(P=1.95 \times$ $10^{-8}$ for CPASSOC analysis; Supplementary Table 2), and was further validated to be associated with the mean volume of hippocampus, one of the earliest affected brain regions in $\mathrm{AD}$, in the Enhancing NeuroImaging Genetics through Meta-Analysis (ENIGMA) consortium cohort comprising MRI images of

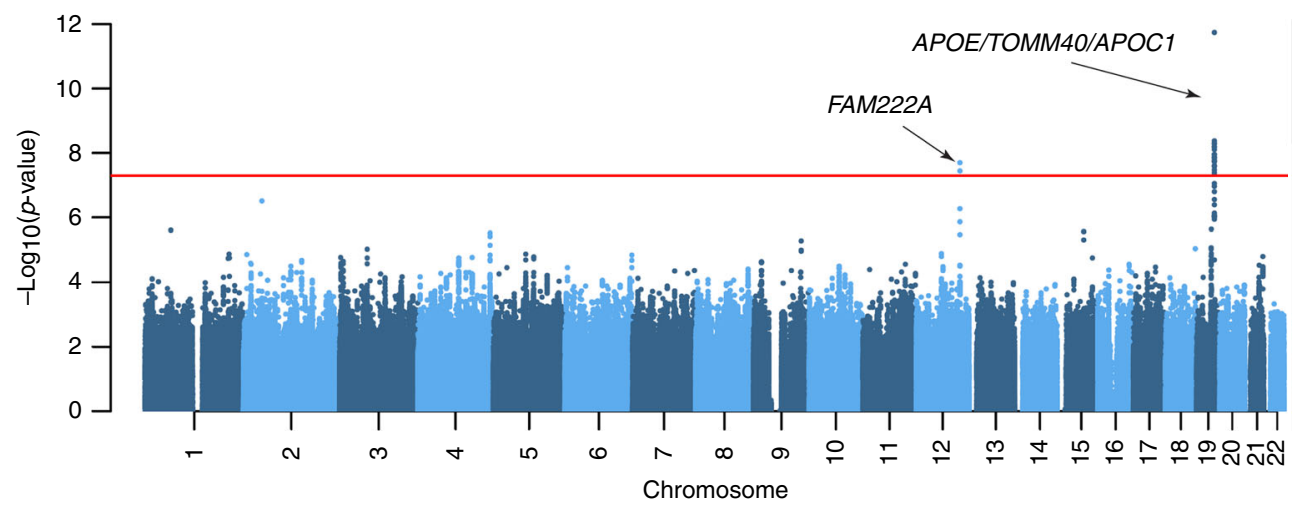

Fig. 1 CPASSOC analysis of the ADNI cohort. Manhattan plot of CPASSOC analysis combining GWAS summary statistics of ROIs in the green-colored module (Supplementary Fig. $1 \mathrm{~m}$ ) including left hippocampus, right hippocampus, basal forebrain, entorhinal area, and planum polare. The red line represents the threshold of $P=5 \times 10^{-8}$ for the genome-wide significance level. Arrows indicate loci associated with regional brain atrophy. Source data are provided as a Source Data file (Source Data for GWAS in Fig. 1). 
30,717 individuals from 50 cohorts $^{20}$ with the same effect direction ( $\beta=38.1, P=8.29 \times 10^{-3}$; Supplementary Table 2$)$.

On a genome-wide scale, FAM222A rs117028417 was only marginally associated with $\mathrm{AD}$ diagnosis in the International Genomics of Alzheimer's Project (I-GAP, $P=0.052)^{21}$. The positron emission tomography (PET) imaging using radiotracer ${ }^{18}$ F-Florbetapir (AV45) provides quantitative measures of amyloid pathology in vivo ${ }^{22}$. To investigate whether SNP rs117028417 is associated with brain $\mathrm{A} \beta$ accumulation, we performed single SNP association test of rs117028417 with AV45 standard uptake value ratio (SUVR) measures for brain regions available in the PET scan dataset from ADNI. The minor allele A of rs117028417 was found significantly associated with longitudinal decrease but not baseline of AV45 SUVR measures in anterior or posterior cingulate $(P<0.0125)$ (Supplementary Table 3), which is relatively consistent with the findings from brain volume associations, where the association direction was positive (i.e. higher volume). rs117028417 is located in an intergenic region that is approximately $5 \mathrm{~kb}$ downstream of FAM222A and $8 \mathrm{~kb}$ downstream of TRPV4. We searched among published GWAS studies of AD or AD-related biomarkers and did not identify common variants previously reported on these two genes. We thus further performed gene-based burden and SKAT tests ${ }^{23,24}$ of coding variants on both FAM222A and TRPV4. All of the coding variants on these two genes available in ADNI whole-genome sequencing data were low-frequency or rare variants with minor allele frequency less than 0.05 (Supplementary Table 4). FAM222A, but not TRPV4, displayed significant association with AV45 SUVR longitudinal changes in anterior or posterior cingulate or lateral parietal regions in the burden test after adjusting for 10 independent tests $(P<0.005)$ (Supplementary Table 5), collectively suggesting a possible role of FAM222A in brain amyloid deposition. However, when we tested rs117028417 for genetic association with AD cerebrospinal fluid (CSF) $A \beta$ and tau biomarkers, only nominal association of rs117028417 with total tau annual change could be discovered, and there was no association with baseline CSF $A \beta$ and tau on both single SNP association tests and variant burden tests (Supplementary Tables 6, 7), indicating that FAM222A variant may not have a strong genetic influence on $\mathrm{AD}$ biomarkers.

FAM222A-encoded protein accumulates within amyloid plaques. The large independent $\mathrm{AD}$ brain imaging dataset including GWAS studies is not available at this time, making it difficult to further validate the genetic link between FAM222A and ADrelated brain atrophy. However, to elucidate the possible pathological role of FAM222A in $\mathrm{AD}$, we carried out experimental validation to focus on its encoded protein, which we designated as Aggregatin. Aggregatin consists of 452 amino acids with a predicted molecular weight of $47 \mathrm{kD}$, and has not yet been characterized. Using a well-characterized specific antibody against Aggregatin (Supplementary Fig. 5a-e), Aggregatin was found predominantly expressed in the central nervous system (CNS) including both the brain and the spinal cord, but not in other tissues such as heart, spleen, lung, kidney, or liver in mice or humans (Supplementary Fig. 5d, e). There was a slight increase in the expression of Aggregatin in brain lysates from $\mathrm{AD}$ patients compared to age-matched control subjects (Supplementary Fig. 5f-h). The most distinct pattern of Aggregatin immunostaining observed in $\mathrm{AD}$ was that Aggregatin was remarkably immunoreactive within the center of amyloid plaques, which were stained by the pan- $\mathrm{A} \beta$ antibodies $6 \mathrm{E} 10$ and $4 \mathrm{G} 8$, the $\mathrm{N}$ terminal truncated and modified pyroglutamate $A \beta$ species $A \beta$ [N3pe] antibody 82E1, fibrillar $A \beta$ dye thioflavin-S (Thio-S) or oligomer $\mathrm{A} \beta$ antibody NU-4 ${ }^{25}$ (Fig. $2 \mathrm{a}, \mathrm{b}$ and Supplementary
Fig. 6a-e). In contrast, all control brain sections lacking detectable amyloid plaques demonstrated weak diffusive Aggregatin immunoreactivity without association with puncta (Fig. 2a).

Robust Aggregatin staining of the central core of amyloid deposits was consistently observed in the brains of multiple mouse models for $\mathrm{AD}$ including $5 \mathrm{XFAD}^{26}, \mathrm{TgCRND}^{27}, \mathrm{APP} /$ $\mathrm{PS}_{1}^{28}, \mathrm{Tg} 2576^{29}$, and $3 \mathrm{xTg}^{30}$ transgenic mice overexpressing human mutant APP along with or without human mutant PS1 (Fig. 2c, d and Supplementary Fig. 6f-h). With the exception of 5XFAD or Tg2576 mice in which Aggregatin-positive foci were connected with wispy fibrils, Aggregatin within amyloid deposits of other transgenic mice showed negligible projecting fibrillar structures, similar as in human plaques. Despite the general localization of Aggregatin large puncta to the core of amyloid deposits, they highly co-localized with $\mathrm{A} \beta$ in $5 \mathrm{XFAD}$ mice but not in $\mathrm{AD}$ patients or TgCRND8 mice, together indicating that the processes contributing to amyloid deposition may be different in human and different animal models. Notably, the formation of Aggregatin puncta occurred concurrently with amyloid deposition, but was not present in the pre-depositing young 5XFAD mice (Supplementary Fig. 6i). The characteristic Aggregatinpositive core staining was abolished by the pre-absorption of primary antibodies with human recombinant Aggregatin protein (rAggregatin) purified by combined $10 \mathrm{~K}$ dialysis and sizeexclusion chromatography, but not $A \beta_{1-42}$ peptides (Supplementary Fig. 7a), further validating the specificity of the antiAggregatin antibody. To confirm the presence of Aggregatin within amyloid deposits, we isolated amyloid cores purified by sucrose density gradient fractionation of $2 \%$ sodium dodecyl sulfate (SDS) homogenized AD or 5XFAD mouse brains. Dot blot and immunoblot studies of proteins under native and denatured forms respectively confirmed the existence of full-length Aggregatin without noticeable cleaved products in the SDSresistant insoluble core-enriched fractions positive for $6 \mathrm{E} 10$ (Fig. 2e-h).

Aggregatin physically interacts with $\mathbf{A} \boldsymbol{\beta}$. The radioimmunoprecipitation assay buffer (RIPA) widely used for coimmunoprecipitation failed to extract Aggregatin from AD brains (Supplementary Fig. 7b), making it difficult to examine the likely association between Aggregatin and $\mathrm{A} \beta$ in $\mathrm{AD}$. To overcome this obstacle, we performed in vitro pull-down assays using synthetic $\mathrm{A} \beta_{1-40}$ or $\mathrm{A} \beta_{1-42}$ and rAggregatin. Dynamic light scatting (DLS), circular dichroism (CD), and SDS-PAGE assays of rAggregatin indicated that rAggregatin existed in the soluble partially folded monomeric state (Supplementary Fig. 7c-e). Notably, rAggregatin co-precipitated with different forms of $A \beta_{1-40}$ or $A \beta_{1-42}$ (Fig. 3a and Supplementary Fig. 7f, g). Consistently, immobilized monomeric $\mathrm{A} \beta_{1-40}$ or $\mathrm{A} \beta_{1-42}$ was also able to pull down rAggregatin (Supplementary Fig. $7 \mathrm{~h}$ ). Further surface binding affinity assays revealed that immobilized $A \beta_{1-40}$ or $A \beta_{1-42}$ bound to rAggregatin, and similarly, immobilized rAggregatin bound to $\mathrm{A} \beta_{1-40}$ or $\mathrm{A} \beta_{1-42}$ all within the nanomolar ranges (Fig. $3 \mathrm{~b}, \mathrm{c}$ and Supplementary Fig. $7 \mathrm{i}, \mathrm{j}$ ). In agreement with these results, surface plasmon resonance (SPR) measurements confirmed that $A \beta_{1-42}$ bound to immobilized rAggregatin at the low nanomolar dissociation equilibrium constant (Kd) (Fig. 3d). Although no measurement was noted in blank or BSA-immobilized sensor chips (Supplementary Fig. 7k), signal spikes produced in the SPR assays may be in proportion to the mass of $\mathrm{A} \beta$ aggregates, making dynamic measurements unlikely consistent with the surface binding affinity assessments at the steady state. To investigate the binding of rAggregatin to $A \beta$ ex vivo, we performed an in situ binding assay in which fixed brain sections of $\mathrm{AD}$ patients or 5XFAD mice were incubated with Flag-tagged rAggregatin and 
a

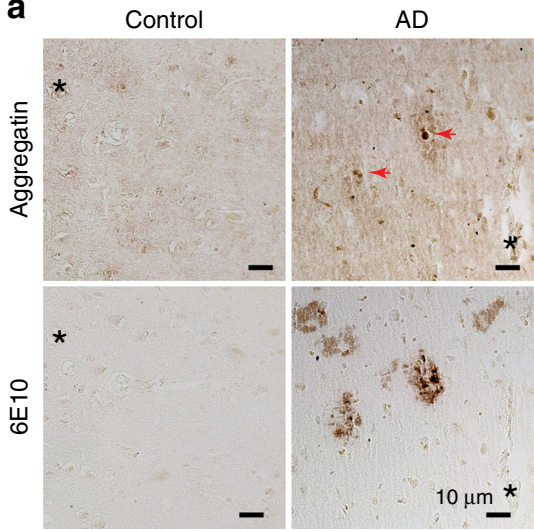

d

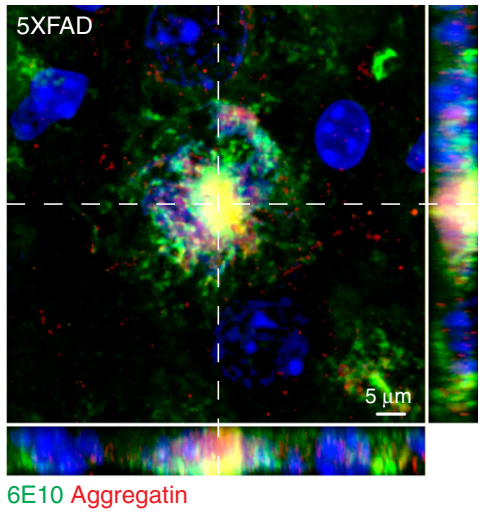

b

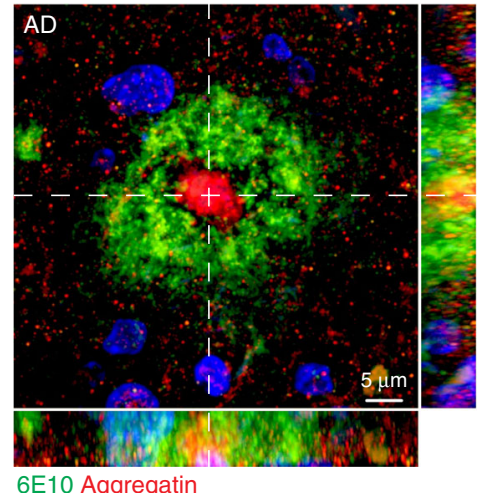

e

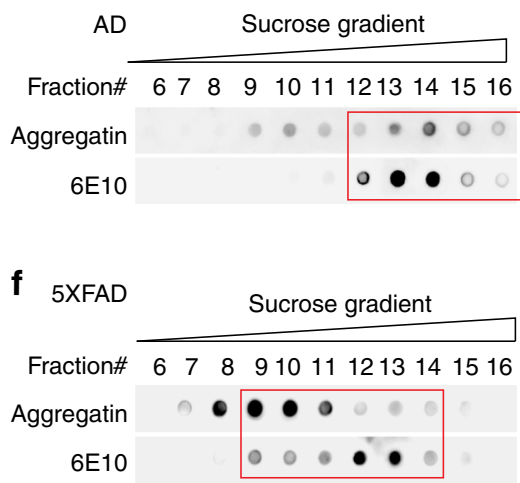

C

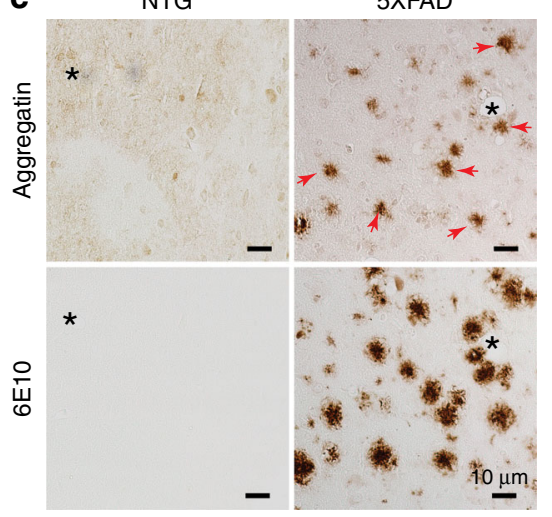

9

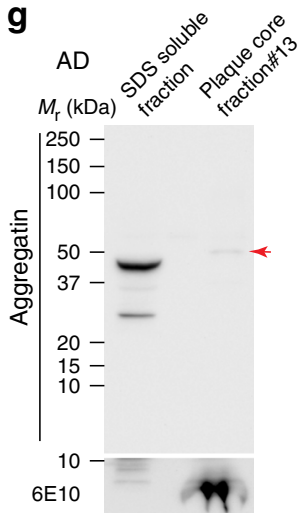

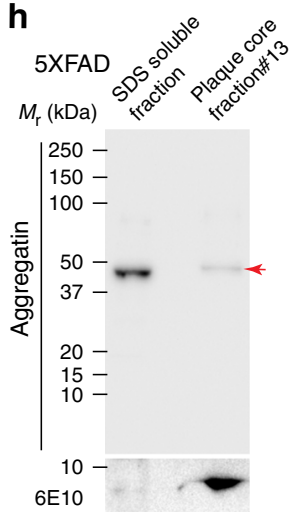

Fig. 2 Aggregatin accumulates within the center of amyloid deposits. a Representative images of immunohistochemistry of Aggregatin (arrowheads) and amyloid plaques (stained by the $6 \mathrm{E} 10$ antibody) in adjacent sections (denoted by asterisks) of cortices of sporadic AD patients. b Representative fluorescent images of Aggregatin (Red), amyloid plaques (Green, stained by the 6E10 antibody) and DAPI nuclei staining (Blue) in cortices of sporadic AD. c Representative images of immunohistochemistry of Aggregatin (arrowheads) and amyloid plaques (stained by the 6E10 antibody) in adjacent sections (denoted by asterisks) of brains of 6-month-old 5XFAD mice. d Representative images of Aggregatin (Red), amyloid plaques (Green, stained by the 6E10 antibody) and DAPI nuclei staining (Blue) in brains of 6-month-old 5XFAD mice. e, $\mathbf{f}$ Representative dot blots of Aggregatin and A $\beta$ (6E10) in serial fractions of amyloid plaques separated by differential centrifugation in sucrose gradient from sporadic AD patients (e) or 6-month-old 5XFAD mice (f). $\mathbf{g}$, $\mathbf{h}$ Representative immunoblots of Aggregatin and A $\beta$ (6E10) in the SDS-resistant insoluble core-enriched fraction from sporadic AD patients (g) or 6-monthold 5XFAD mice (h). Arrow heads point Aggregatin. Due to the presence of urea used for plaque core protein extraction, plaque core fractions show slight shifts compared to SDS soluble fraction. All experiments were independently performed at least three times. Source data are provided as a Source Data file (Source Data for Statistics and Blots).

stained by an anti-Flag antibody. Remarkably, all amyloid deposits were labelled by rAggregatin (Supplementary Fig. 7l-n). Considering the widespread presence of $\mathrm{A} \beta$ in brains, it was not surprising that brain sections also showed background staining after rAggregatin incubation. Notably, amyloid deposits and the background binding of rAggregatin were completely abolished by pre-incubation of rAggregatin with $A \beta_{1-40}$ or $A \beta_{1-42}$ (Supplementary Fig. 7l-n), confirming that rAggregatin binds amyloid deposits by interacting with $\mathrm{A} \beta$. Collectively, these results highlight the pathological relevance of Aggregatin in $\mathrm{AD}$, and show that Aggregatin is a $\mathrm{A} \beta$ binding protein with high-affinity.

Aggregatin binds to $\mathbf{A} \boldsymbol{\beta}$ via its $\mathbf{N}$-terminal region. Next, we generated a series of rAggregatin deletion mutants to map the binding region for $A \beta$. Although rAggregatin alone does not form oligomers or aggregates, the composition of $\mathrm{A} \beta$ preparations at the micromolar range quickly changes over time due to the formation of higher order oligomers, which are expected to influence the Aggregatin and $A \beta$ interaction. To quantitatively identify the binding strength of different rAggregatin deletion mutants, the in situ binding assay rather than pull-down assay was used for the binding motif mapping. The deletion of residues from 1 to 80 (designated as NABD, N-terminal A $\beta$ binding domain), but not residues outside of this region, was found to greatly reduce the binding of rAggregatin to amyloid deposits (Fig. 3e-g and Supplementary Fig. 8a, b). Recombinant NABD (rNABD) alone was able to bind to amyloid deposits or $A \beta_{1-42}$ similar as full-length rAggregatin, and caused a dose-dependent decrease in the association between rAggregatin and amyl deposits when coincubated (Fig. 3b, c, e-g and Supplementary Fig. 8c, d), together suggesting NABD as the domain both necessary and sufficient for $\mathrm{A} \beta$ binding. The residues from 61 to 80 appear to be a core motif for NABD though they alone were not sufficient to bind amyloid deposits (Fig. 3e-g and Supplementary Fig. 8a, b). Notably, rNABD bound amyloid deposits in a length-dependent manner, and rAggregatin with partial deletions of every 5 amino acids within the core motif of NABD exhibited weaker but still strong interactions with amyloid deposits (Supplementary Fig. 8a, b), further indicating that NABD may contain multiple sites cooperatively involved in $A \beta$ binding.

Aggregatin cross-seeds $\mathbf{A} \boldsymbol{\beta}$ via direct binding. Given the strong interaction between Aggregatin and $A \beta$, we further set out to determine whether Aggregatin would influence the $A \beta$ 


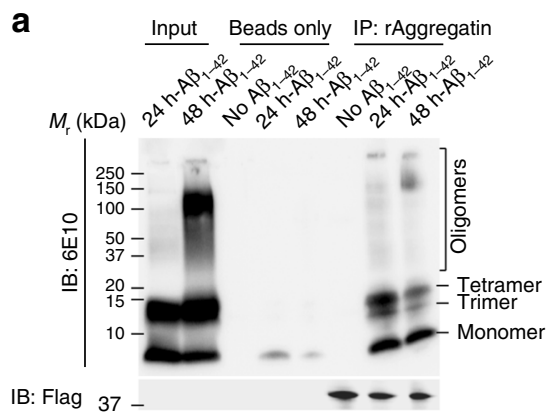

IB: Flag 37 - b

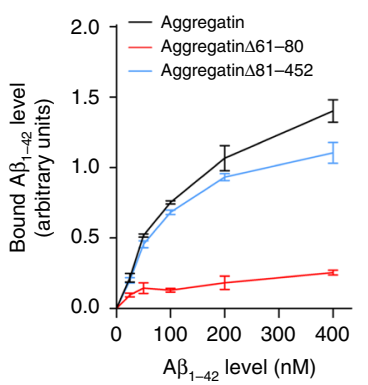

C

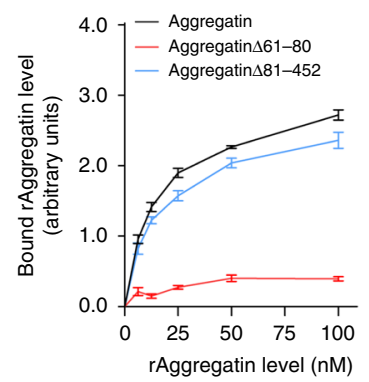

d

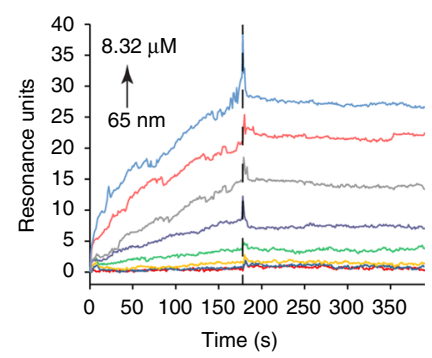

e

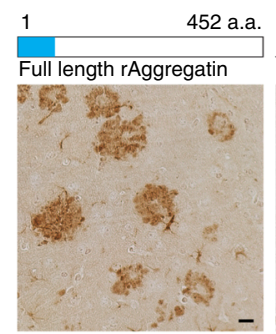

160

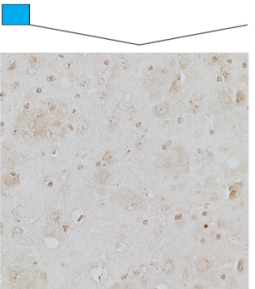

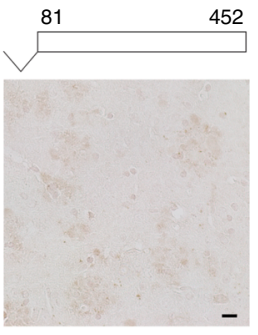

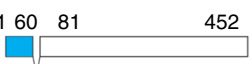

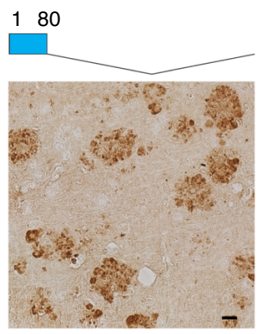

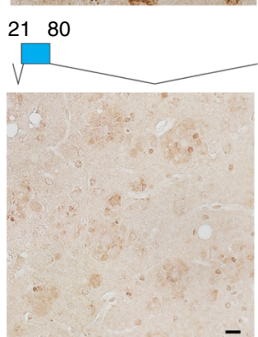

170

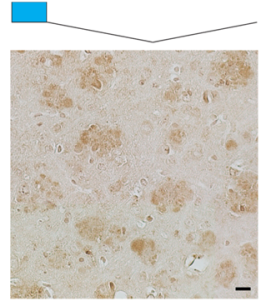

3180
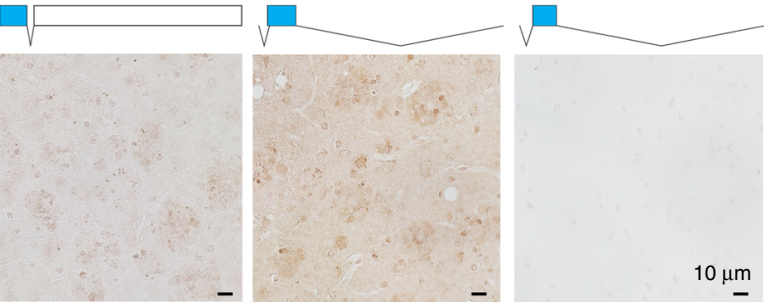

f

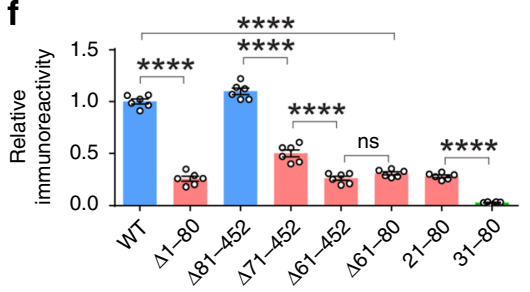

g

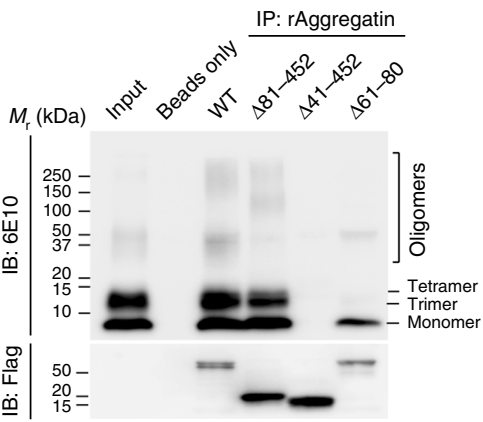

Fig. 3 Aggregatin interacts with A $\boldsymbol{\beta}$. a Coimmunoprecipitation of purified Flag-tagged rAggregatin and $A \beta_{1-42}$ (pre-aggregated in vitro for 24 or 48 h). rAggregatin was immunoprecipitated using streptavidin magnetic beads and immunoblotted using the antibody to Flag. $\mathbf{b}$ Measurement of $A \beta_{1-42}$ levels bound to immobilized $r$ Aggregatin (normalized to maximal $r$ Aggregatin and $A \beta_{1-42}$ binding). $n=3$ independent experiments. c Measurement of rAggregatin levels bound to immobilized $A \beta_{1-42}$ (normalized to maximal $r A g g r e g a t i n$ and $A \beta_{1-42}$ binding). $n=3$ independent experiments. $\mathbf{d}$ Bio-layer interferometry measurement of the binding kinetics of monomeric $A \beta_{1-42}$ to immobilized $r$ Aggregatin. Curves are corresponded to $A \beta_{1-42}$ at 8320 , 4160 , $2080,1040,520,260,130$ and $65 \mathrm{nM}$ from the top to bottom. e, f Representative immunohistochemistry (e) and quantification (f) of rAggregatin immunoreactivity (Flag antibody) in 5XFAD mouse brain sections after incubation with $100 \mathrm{nM}$ indicated rAggregatin deletion mutants ( $n=6$ independent experiments in each group). Blocks with blue color on the top of each immunohistochemistry image show NABD. $\mathbf{g}$ Coimmunoprecipitation analysis of purified Flag-tagged $r$ Aggregatin deletion mutations and $A \beta_{1-42}$ using streptavidin magnetic beads. Source data are provided as a Source Data file (Source Data for Statistics and Blots). Data are means \pm s.e.m ( \pm is the plus-minus sign). One-way analysis of variance (ANOVA) followed by Tukey's multiple comparison test. ${ }^{\star \star \star \star} P<0.0001$. ns, non-significant.

aggregation process. $A \beta$ aggregation kinetics were first monitored in vitro using $A \beta_{1-40}$ or $A \beta_{1-42}$ for the thioflavin $T(T h T)$ based fluorescence assay. As illustrated by changes in ThT-associated fluorescence, $A \beta$ self-aggregated only at high concentrations whereas rAggregatin alone did not produce any observable aggregate (Fig. 4a and Supplementary Fig. 9a). Remarkably, once co-incubated with $r$ Aggregatin, $A \beta$ was able to form aggregates at low concentrations even in the nanomolar range (Fig. $4 \mathrm{a}, \mathrm{b}$ and Supplementary Fig. 9a-c). With increasing concentrations of rAggregatin, the lag times of the aggregation reaction were greatly decreased (Fig. 4a and Supplementary Fig. 9b). As a control, rAggregatin $\Delta 61-80$ had similar folding as wild type rAggregatin, but failed to induce $A \beta_{1-42}$ aggregation (Fig. 4a and Supplementary Fig. $7 \mathrm{~d}$, e). These observations were confirmed using immunoblot and dot blot analyses for $A \beta$ aggregation measurements under denatured and native conditions, which showed that Aggregatin but not rAggregatin $\Delta 61-80$ indeed promoted $A \beta_{1-42}$ oligomerization (Fig. 4c-f and Supplementary Fig. 9d). Of note, due to the sensitivity of immunoblot, $A \beta$ oligomer was only detectable with long exposure when $A \beta_{1-42}$ at the low micromolar but not nanomolar was applied. Consistently, transmission electron microscopy analyses revealed that soluble $A \beta_{1-42}$ protofibrils ${ }^{31}$ were more abundant and have more complicated structures in the presence of rAggregatin during the early phase of incubation when $A \beta$ fibrils were absent (Fig. 4g). As expected, the low concentration of $A \beta_{1-42}$ only yielded very few short and un-branched fibrils after long periods of incubation under negative staining (Fig. 4g), and rAggregatin alone did not form identifiable particles or large aggregates (Supplementary Fig. 9e). Strikingly, co-incubation of low micromolar $A \beta_{1-42}$ with $\mathrm{rAg}$ gregatin lead to the formation of large micrometer-long branched fibrils (Fig. 4g and Supplementary Fig. 9f), which were Thio-Spositive and visible under the fluorescent microscopy (Fig. 4h). Taken together, these data imply Aggregatin as a potent seeding factor for $A \beta$ oligomerization and aggregation.

Aggregatin regulates amyloid deposition. A $\beta$ levels are low in young especially predepositing 5XFAD mice ${ }^{26}$. To examine the effect of extracellular Aggregatin on amyloid deposition with 
a

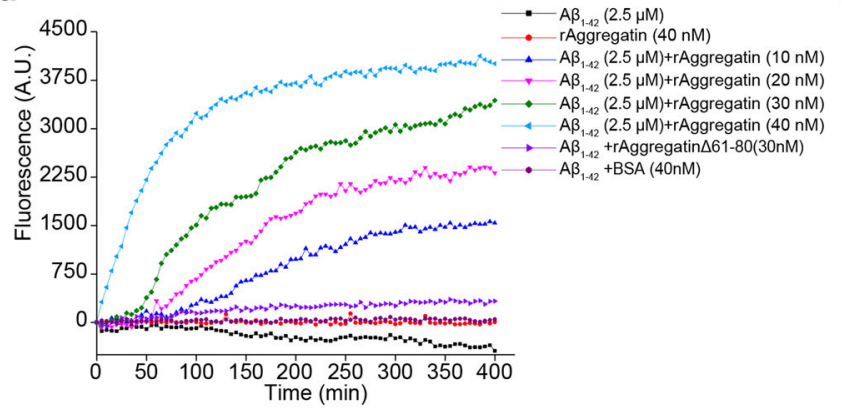

b

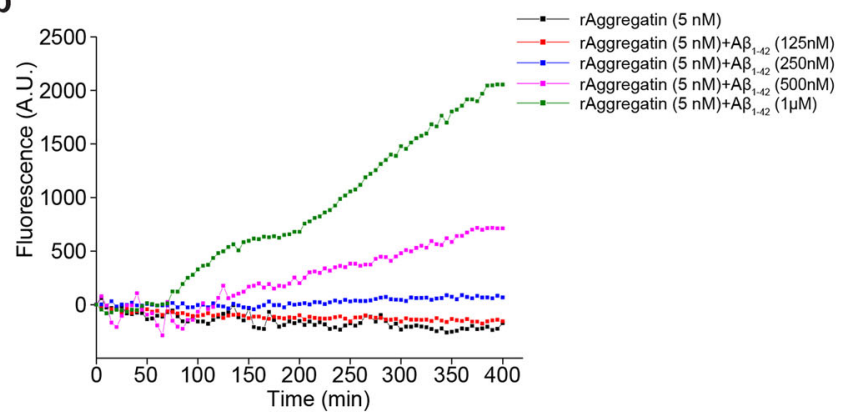

C

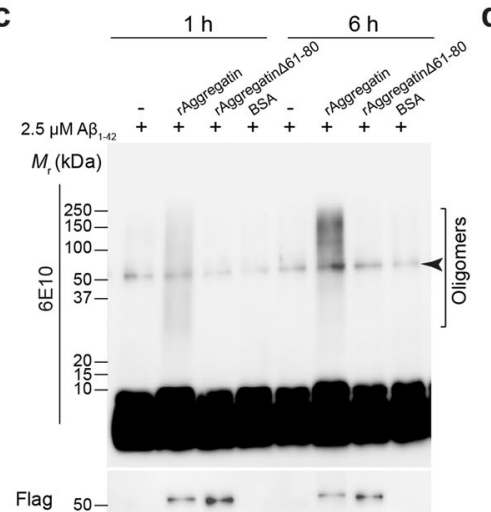

g
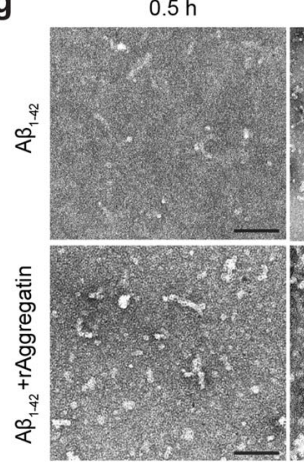

$6 \mathrm{~h}$
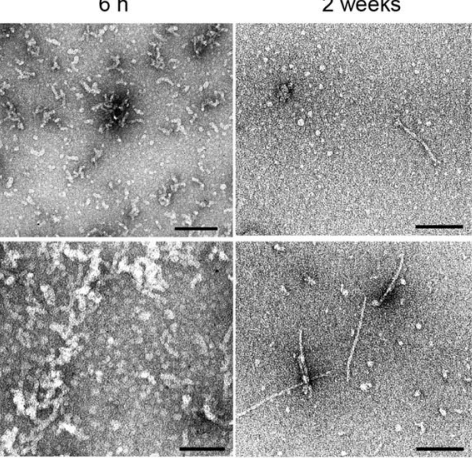

d

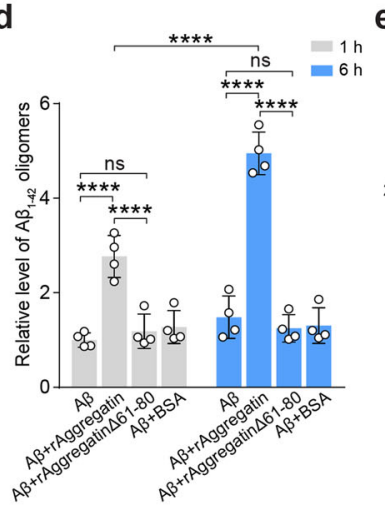

e

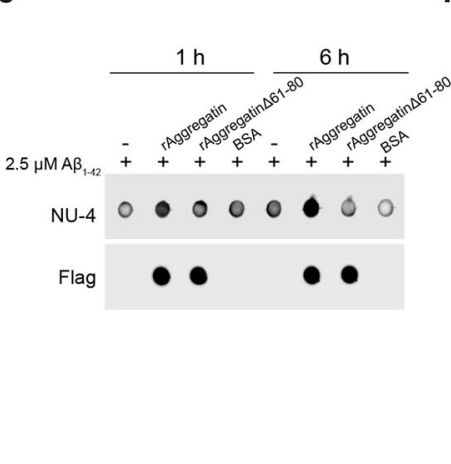

f

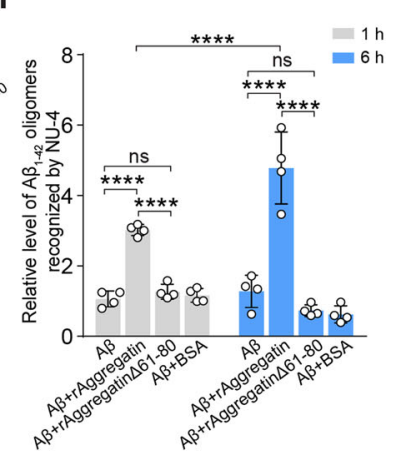

4 weeks
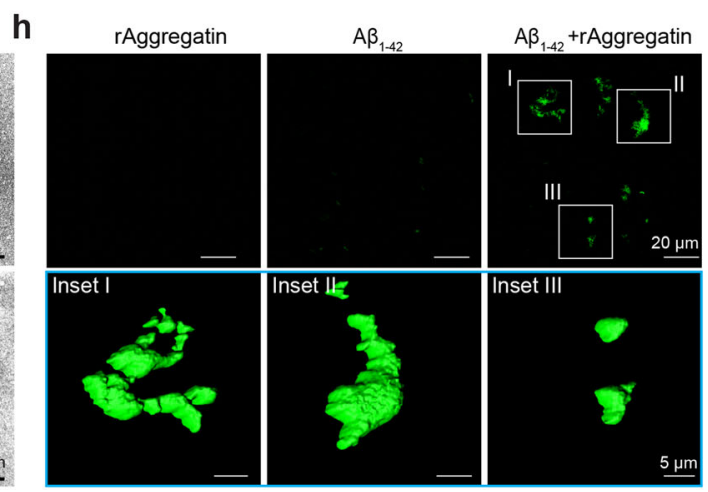

Fig. 4 Aggregatin accelerates $\mathbf{A} \boldsymbol{\beta}$ aggregation in vitro. a ThT-based assay measuring aggregation kinetics of $2.5 \mu \mathrm{M} A \beta_{1-42}$ in the presence of various concentrations of rAggregatin ( $n=5$ independent experiments in each time points). $\mathbf{b}$ ThT-based assay measuring aggregation kinetics of various concentrations of $A \beta_{1-42}$ in the presence of $5 \mathrm{nM}$ rAggregatin ( $n=5$ independent experiments in each time points). c, $\mathbf{d}$ Representative immunoblot (c, light exposure shown in Fig. S9d) and quantification (d) of $A \beta_{1-42}$ oligomers recognized by $6 \mathrm{E} 10$ in the $30 \mathrm{nM}$ rAggregatin and $2.5 \mu \mathrm{M} A \beta_{1-42}$ mixture collected after 6 -h co-incubation ( $n=4$ independent experiments). Arrow head points to non-specific bands due to long exposure. $\mathbf{e}, \mathbf{f}$ Representative dot blot $(\mathbf{e})$ and quantification (f) of $A \beta_{1-42}$ oligomers recognized by the oligomer $A \beta$ specific antibody NU-4 in the $30 \mathrm{nM} r A g g r e g a t i n$ and $2.5 \mu \mathrm{M} A \beta_{1-42}$ mixture collected after 6-hour co-incubation ( $n=4$ independent experiments). $\mathbf{g}$ Negative staining electron microscopy of $2.5 \mu \mathrm{M}$ A $\beta_{1-42}$ aggregates after 0.5-h, 6-h, 2-week, and 4-week co-incubation with or without $30 \mathrm{nM}$ rAggregatin. h Representative 3D images of $2.5 \mu \mathrm{M} A \beta_{1-42}$ aggregates stained by Thio-S after 4-week co-incubation with or without $30 \mathrm{nM}$ rAggregatin. Source data are provided as a Source Data file (Source Data for Statistics and Blots). Data are means \pm s.e.m. One-way analysis of variance (ANOVA) followed by Tukey's multiple comparison test. ${ }^{\star * \star \star} P<0.0001$. ns, non-significant.

unrestricted access to predeposit-state $\mathrm{A} \beta$, we performed intracerebroventricular (ICV) infusion of Flag-tagged rAggregatin or rAggregatin $\triangle 61-80$ into 5XFAD mice at 4-month-old, when $\mathrm{A} \beta$ rises to high levels ${ }^{26}$ (Supplementary Fig. 10a). Infusion did not cause the death of mice or histological abnormalities in the brain. Importantly, the levels of total A $\beta$, APP or BACE1 remained unchanged 4 weeks after rAggregatin infusion, indicating that rAggregatin did not affect $A \beta$ production or degradation (Supplementary Fig. 10b, c). ICV infused rAggregatin was detected in amyloid deposit (Fig. 5a). Remarkably, compared to age-matched control mice infused with artificial cerebrospinal fluid (aCSF), rAggregatin-infused mice showed greatly increased amyloid deposition spreading the brain at 5 months of age, which could be completely blocked by the deletion of NABD core motif (Fig. 5b, c and Supplementary Fig. 10d, e). As prominent AD pathological features, microgliosis and astrogliosis are closely associated with amyloid deposits in 5XFAD mice ${ }^{26,32}$. Corresponding to increased plaque load, 5XFAD mice infused with rAggregatin but not rAggregatin $\triangle 61-80$ exhibited more microgliosis and astrogliosis compared to aCSF-infused control 5XFAD mice (Fig. 5d and Supplementary Fig. 10f). 5XFAD mice begin to show cognitive deficits at around 4-months-old ${ }^{33,34}$. Compared with NTG mice, FAD mice exhibited significantly impaired Y-maze and Barnesmaze performance, both of which were significantly exacerbated in transgenic mice with rAggregatin but not rAggregatin $\Delta 61-80$ infusion (Fig. 5e, f). To further examine the role of neuronal 
a
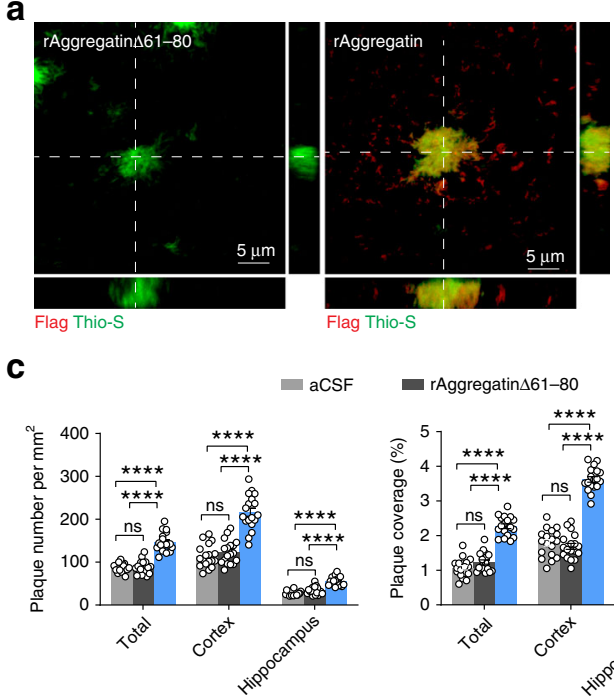

C

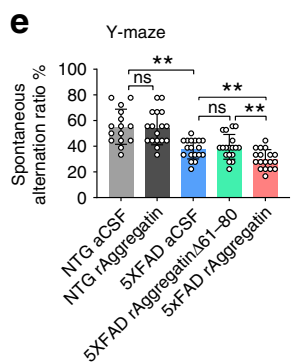

f
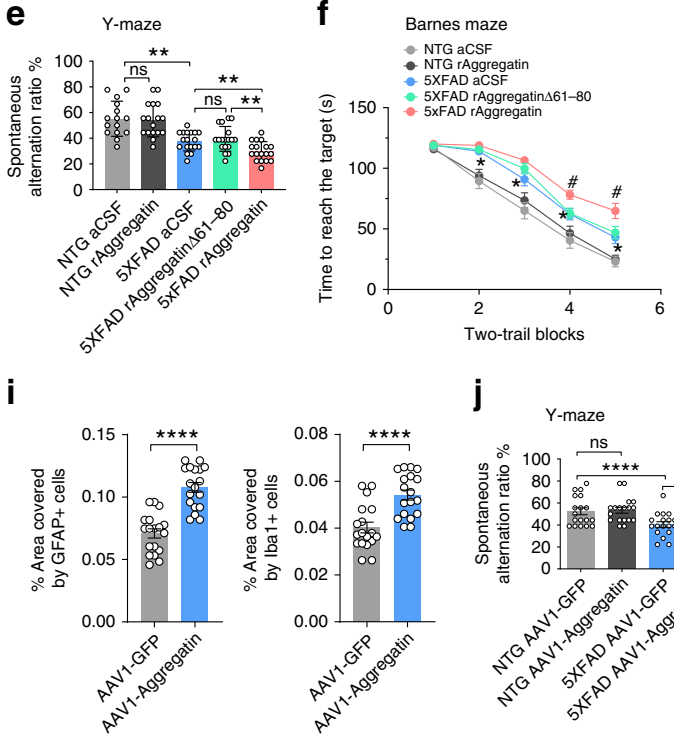

j

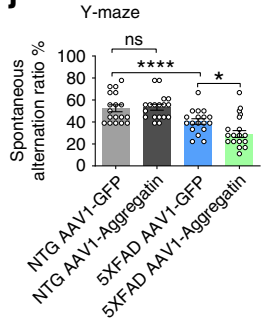

g

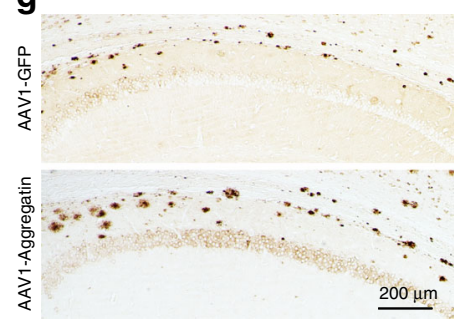

h
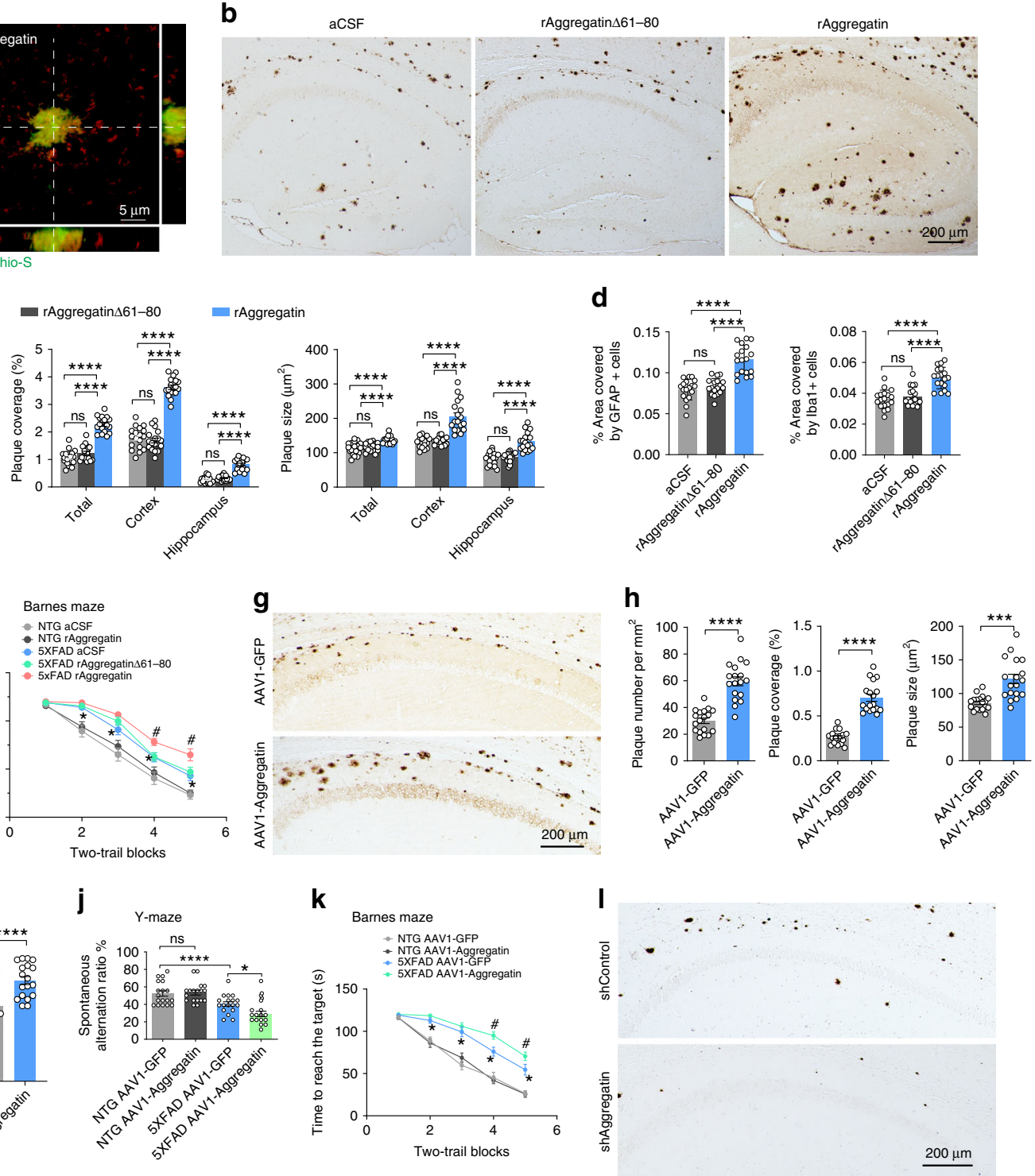

m

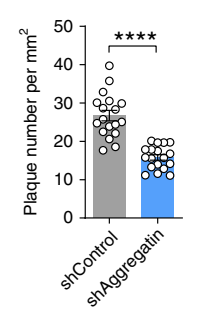

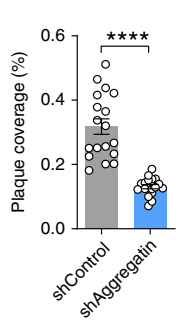
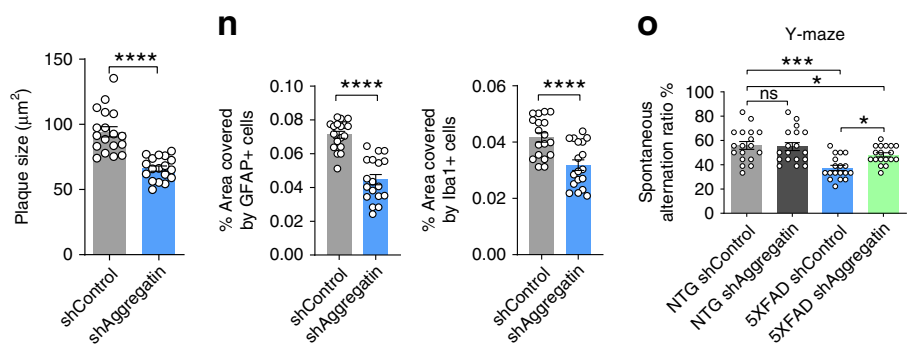

Fig. 5 Aggregatin regulates amyloid deposits. 5-month-old 5xFAD mice were ICV infused with Flag-tagged rAggregatin $\Delta 61-80$ or rAggregatin for 4 weeks. a Representative images of Flag-tagged Aggregatin (Red) and amyloid plaques (Green, Thio-S) in the brain. b, c Representative images (b) and quantification (c) of plaque by NU-4 antibody in the total brain (Total), cortex or hippocampus ( $n=18$ mice in each group). d Quantification of astrogliosis and microgliosis in hippocampus (representative images shown in Supplementary Fig. 10f). e, f Y-maze (e) and Barnes maze (f) performance ( $n=15,17$, 18, 18, and 18 mice for NTG aCSF, NTG rAggregatin, 5XFAD aCSF, 5XFAD rAggregatin $\triangle 61-80$, and 5XFAD rAggregatin respectively). 5-month-old 5XFAD mice were injected with AAV1-GFP or AAV1-Aggregatin at 1.5 month-old. $\mathbf{g}$, $\mathbf{h}$ Representative images ( $\mathbf{g}$ ) and quantification (h) of plaques stained by NU-4 in the hippocampus ( $n=18$ mice in each group). $\mathbf{i}$ Quantification of astrogliosis and microgliosis in the hippocampus $(n=18$ mice in each group). $\mathbf{j}, \mathbf{k}$ Ymaze (j) and Barnes maze (k) performance ( $n=18$ mice in each group). Representative images are shown in Supplementary Fig. 11g. 5-month-old 5XFAD mice were injected with AAV1-shControl or AAV1-shAggregatin at 1.5-month-old. I, $\mathbf{m}$ Representative images (h) and quantification (i) of plaques stained by NU-4 in the hippocampus ( $n=18$ mice in each group). $\mathbf{n}$ Quantification of astrogliosis and microgliosis in the hippocampus ( $n=18$ mice in each group). Representative images are shown in Supplementary Fig. 12f. o, p Y-maze (o) and Barnes maze (p) performance ( $n=18$ mice in each group). Source data are provided as a Source Data file (Source Data for Statistics and Blots). Data are means \pm s.e.m. Student's $t$-test or one and two-way analysis of variance (ANOVA) followed by Tukey's multiple comparison test. ${ }^{\star} P<0.05,{ }^{\#} P<0.05$ (relative to aCSF, AAV1-GFP or shControl AAV1), ${ }^{\star \star \star \star} P<0.001,{ }^{\star \star \star \star} P<$ 0.0001. ns, non-significant. 
Aggregatin in amyloid deposition, we injected adeno-associated virus serotype 1 encoding human Aggregatin or GFP alone under the neuron specific promoter eSYN (AAV1-Aggregatin or AAV1GFP) into the hippocampus CA1 of young predepositing 5XFAD mice at 1.5-month-old (Supplementary Fig. 11a). When analyzed at 5 months of age, in line with ICV infusion experiments, intrahippocampal injection of AAV1-Aggregatin significantly increased amyloid deposition without any effect on total A $\beta$ levels in the GFPpositive hippocampal region, but not in the brain areas without AAV1-Aggregatin delivery (Fig. $5 \mathrm{~g}, \mathrm{~h}$ and Supplementary Fig. 11b-f), together suggesting that Aggregatin is sufficient to enhance amyloid deposition in vivo. Consistently, amyloid deposition associated microgliosis, astrogliosis, and cognitive deficits were also worsened by neuronal Aggregatin overexpression (Fig. 5i-k and Supplementary Fig. 11g). To investigate whether Aggregatin was required for amyloid deposition, we performed intrahippocampal injection of AAV1 co-expressing GFP and a short hairpin RNA targeting Aggregatin (AAV1-shAggregatin) or control shRNAi (AAV1-shControl) in predepositing 5XFAD mice (Supplementary Fig. 5b, c). It was observed that decreasing Aggregatin was not associated with neuronal loss or altered total A $\beta$ (Supplementary Fig. 12a). At 5 months of age, the injection of AAV1-shAggregatin significantly alleviated amyloid deposition in the GFP-positive areas of hippocampus compared to AAV1shControl injection, but not in the GFP-negative brain areas (Fig. 5l, $\mathrm{m}$ and Supplementary Fig. 12b-e). Likewise, Aggregatin reduction significantly alleviated amyloid deposit associated microgliosis, astrogliosis, and cognitive impairment (Fig. 5n-p and Supplementary Fig. 12f). Taken together, these results further imply that Aggregatin is also an important factor necessary for amyloid deposition.

\section{Discussion}

Here, we report on Aggregatin, the protein encoded by $F A M 222 A$, as a plaque core protein directly binding $A \beta$ and facilitating $A \beta$ aggregation, a process thought to be central in $\mathrm{AD}$ onset. Therefore, this work provides strong experimental evidence supporting a pathophysiological role for Aggregatin in AD.

In people diagnosed with $\mathrm{AD}$ or mild cognitive impairment (MCI), a proportion of whom can progress to $\mathrm{AD}, F A M 222 \mathrm{~A}$ is associated with the module enriched for atrophy in AD-affected brain regions. FAM222A association with hippocampal volume could be validated in the replication ENIGMA cohort, together pointing to a potential mechanism by which FAM222A may affect regional brain atrophy. Notably, our cross phenotype association analysis also led to the identification of long-established $\mathrm{AD}$ risk genes APOE, TOMM40, and APOC1 exclusively in the same module, suggesting possible genetic interplays between FAM222A and $\mathrm{AD}$ risking genes. Interestingly, although we only discovered marginal association between rs117028417 and AD diagnosis, FAM222A, but not the nearby gene TRPB4, was found significantly associated with longitudinal increase of brain amyloid deposition. Along this line, as $\mathrm{AD}$ is a genetically complex and multifactorial disease with different etiological subtypes, FAM222A variants or pathogenic mutations strongly associated with $\mathrm{AD}$ may be present in subsets of $\mathrm{AD}$ patients. Nevertheless, although our genetic discovery study did not observe a strong influence of FAM222 variant on $\mathrm{AD}$ risk and biomarkers, the module enriched for FAM $222 \mathrm{~A}$ and previously reported $\mathrm{AD}$ risk variants likely represents a statistical AD-specific cluster worthy of further investigation using independent $\mathrm{AD}$ neuroimaging databases.

Due to the limited sample size, only a slight, but not significant increase of Aggregatin mRNA level was observed in AD cortices collected in our laboratory (Supplementary Fig. 13a, b). However, analysis of previously published microarray data of the Mount
Sinai Brain Bank (MSBB) cohort ${ }^{35}$ found significantly increased levels of Aggregatin mRNA in many brain regions especially cortices of $\mathrm{AD}$, which were also tested to be associated with $\mathrm{AD}$ neuropathologies (Supplementary Fig. 13c, d and Supplementary Tables 8,9$)$. DNA methylation is one of several epigenetic mechanisms regulating gene expression ${ }^{36}$ and has been implicated in $\mathrm{AD}^{37}$. Interestingly, several methylation sites on FAM222A associated with AD could be identified (Supplementary Fig. 14 and Supplementary Table 10), indicating the likely involvement of FAM222A in AD pathogenesis through epigenetic regulation of its gene expression. Considering Aggregatin mRNA levels were usually measured at one time-point, future studies may be interesting to investigate longitudinal changes of Aggregatin gene expression and their relationship with neuropathologies during the progression of disease. While the relationship between FAM222A DNA methylation, transcription, translation, and posttranslational modification remains to be determined, these interesting findings provide further genetic evidence supporting the association of FAM 222A to AD.

Consistent with the genetic association of FAM222A with longitudinal brain $A \beta$ deposition, pathologically accumulated Aggregatin, the protein encoded by FAM222A, is readily noted in plaques in $\mathrm{AD}$ and amyloid deposits in multiple APP transgenic mice, strongly illustrating the pathological function of Aggregatin. Of note, there are remarkable differences in the morphology of Aggregatin puncta and their co-localization with A $\beta$. Similarly, as plaques in AD patients are more complex structures than amyloid deposits in APP transgenic mice ${ }^{38}$, it could be expected that Aggregatin is also present differentially in amyloid core-enriched fractions from $\mathrm{AD}$ patients and 5XFAD mice. A number of explanations may account for the discrepancy regarding the pattern of Aggregatin puncta or presence of Aggregatin in plaques, including but not limited to differences in disease stages, the effects of $A \beta$ clearance and degradation pathways or the length of time spent for plaque deposition. This notion is indeed supported by the observation that while only one or several condensed Aggregatin foci were present in single plaque in $\mathrm{AD}$, amyloid deposits in cortex from patients with Down's syndrome (DS), a complex genetic abnormality developing AD-like pathology, were largely associated with multiple foci (Supplementary Fig. 15).

It is still unclear how Aggregatin becomes accumulated within the center of plaques without the ability for self-aggregation. Aggregatin appears to bind $A \beta_{1-40}$ and $A \beta_{1-42}$ with different affinities. Along this line, amyloid plaques are made up of different $\mathrm{N}$ or $\mathrm{C}$-terminally truncated and modified $\mathrm{A} \beta$ species ${ }^{39}$. Interestingly, we found that Aggregatin was present in exosomes (Supplementary Fig. 16). Although Aggregatin has no signal sequence and is not predicted to be secreted, this data supports the possibility that Aggregatin can be exported into the interstitial fluid. Of note, the presence of exogenously expressed Aggregatin in exosomes of cultured cells is physiologic. There may be other mechanisms responsible for Aggregation secretion under pathological conditions. As Aggregatin protein levels were upregulated in $\mathrm{AD}$, there may be a complex interplay among $\mathrm{A} \beta$ specific forms, Aggregatin expression, post-translational modification, extracellular secretion, and other unknown factors responsible for this. Nevertheless, on the basis of the facts that Aggregatin puncta appear concurrently with amyloid plaques and does not exist in the predepositing mice, Aggregatin should accumulate in plaques before or concurrent with rather than after the well formation of plaques. Aggregatin did not form intraneuronal accumulation in $\mathrm{AD}$ patients and 5XFAD mice. Not surprisingly, we did not observe the presence of Aggregatin puncta in neurons bearing neurofibrillary tangles (Supplementary Fig. 17a). Along this line, intraneuronal APP and/or A $\beta$ immunoreactivity assessed by $6 \mathrm{E} 10$ was not changed by Aggregatin in 5XFAD mice. Therefore, 
Aggregatin may not be involved in intraneuronal protein aggregation. Noteworthily, Aggregatin does not physically interact with tau and other previously reported plaque-associated proteins such as $a$-synuclein and APOE (Supplementary Fig. 17b-d), further implicating the likely specific link between Aggregatin and $A \beta$. However, as $\mathrm{AD}$ is a multifactorial disease, further detailed investigation will still be needed to determine the spatiotemporal relationship between Aggregatin and other $\mathrm{AD}$-related pathologies especially considering the presence of Aggregatin immunoreactivity outside of plaques.

Aggregatin facilitates $A \beta$ aggregation in vitro although it is not clear whether Aggregatin influences the primary or secondary nucleation. Increasing Aggregatin enhances, whereas reduced Aggregatin suppresses amyloid deposition and associated neuroinflammation and cognitive deficits. Of note, in addition to exacerbate $\mathrm{A} \beta$ pathology in adult 5XFAD mice, Aggregatin infusion causes further amyloid deposition in aged 5XFAD mice when amyloid deposit size and number largely plateau (Supplementary Fig. 18). Therefore, Aggregatin is likely an unrecognized co- or even limiting factor both necessary and sufficient for $A \beta$ aggregating into the fibrils to form plaques. Although the bioinformatics analysis of Aggregatin amino acid sequence reveals that Aggregatin does not contain any known conserved functional motifs, our CD characterization of Aggregatin indicated it as at least a partially folded protein containing $\alpha$-helix, $\beta$-sheet, and intrinsically disordered element(s) (Supplementary Fig. 7e). While the structure and physiological function of Aggregatin is still under investigation, we found that Aggregatin was exclusively expressed in the CNS. The substantial loss of Aggregatin in hippocampus does not cause neuronal death, suggesting that Aggregatin may not be vital for neuronal survival. Future studies may be feasible to generate mice with global or neuronal specific deletion of Aggregatin to test whether the knockout of Aggregatin is sufficient to completely abolish amyloid deposition and further validate the pathological role of Aggregatin in amyloid plaque formation and disease progression.

The genetic inhibition of Aggregatin-A $\beta$ interaction was able to suppress Aggregatin-induced $A \beta$ aggregation or amyloid deposits, suggesting that Aggregatin should directly interact with $A \beta$ to regulate its pathology. Of note, although rNABD (i.e. Aggregatin 1-80 or Aggregatin $\Delta 81-452$ ) alone is able to bind $A \beta$, it does not induce $\mathrm{A} \beta_{1-42}$ aggregation or promote amyloid deposits (Supplementary Fig. 19), suggesting that the C-terminal fragment is also required for Aggregatin-induced $A \beta$ aggregation and plaque formation. The exact mechanism for Aggregatin-mediated $A \beta$ aggregation is still under investigation. Noteworthily, likely due to the high $\mathrm{A} \beta$ binding affinity of Aggregatin, the specific antiAggregatin antibody used in this study does not dissociate the Aggregatin- $A \beta$ interaction or prevent Aggregatin-induced aggregation of $A \beta$, and likewise, ICV infusion of the specific antiAggregatin antibody failed to alleviate $\mathrm{A} \beta$ pathologies in 5XFAD mice. Thus, the Aggregatin targeted immunotherapy for AD may require the generation of high-affinity monoclonal antibodies. The genetic manipulation or infusion of Aggregatin did not change APP or A $\beta$ levels, suggesting that Aggregatin is unlikely involved in $A \beta$ production or degradation. However, even though Aggregatin large puncta do not overlap with neurons, microglia or astrocytes, our results cannot rule out the possibility that Aggregatin may regulate amyloidosis indirectly through neuronal function or microglial or astrocytic Aggregatin, which is also worthy of further clarification. APOE4 is the strongest genetic risk factor for LOAD. Although the involvement of APOE in A $\beta$ metabolism might complicate the interpretation of data ${ }^{40}$, future studies will also be interesting to investigate Aggregatin-mediated amyloidosis in vivo on the human ApoE knock-in or ApoE knockout background as previously reported ${ }^{41}$.
In conclusion, we have reported FAM222A as a likely gene associated with $\mathrm{AD}$-related regional brain atrophy, which encodes an amyloid plaque core protein pathologically involved in $A \beta$ assembly and amyloid deposition. Our findings therefore not only inform future genetic studies of FAM222A, but also encourage detailed pathophysiological investigation of its encoded Aggregatin for $\mathrm{AD}$ and related dementia.

\section{Methods}

Samples, genotyping, and imputation. Data used in the preparation of this article were obtained from the ADNI database (http://www.loni.ucla.edu/ADNI). The Illumina SNP genotyping data, demographic information, APOE genotype and baseline diagnosis information from 754 ADNI-1 participants, including 213 cognitive normal individual controls, $175 \mathrm{AD}$ patients, and 366 patients with mild cognitive impairment (MCI) were downloaded from ADNI database. All participants provided written informed consent and study protocols were approved by participating sites' Institutional Review Board.

SNP genotyping of 620,901 markers on ADNI-1 participants were generated using Illumina BeadStudio 3.2 software from bead intensity data. All SNP genotypes are publicly available for download at the ADNI website. For genotype imputation analysis, only SNPs fulfilling the following criteria were included (1) per-SNP call rate $\geq 0.98$; (2) minor allele frequency (MAF) $\geq 0.01$; (3) $P$-value for Hardy-Weinberg equilibrium (HWE) $\geq 10^{-6}$ in our sample set. Imputation was performed using the software MACH-ADMIX ${ }^{42}$ using the 1000 Genomes Project Phase 3 V.5 (http://www.internationalgenome.org) as a reference panel. We excluded SNPs with $\mathrm{R}^{2}<0.3, \mathrm{MAF}<0.01$ and all INDELs from the imputed genotype data to obtain genotypes for 7,512,167 SNPs for subsequent association analyses.

MRI analysis and extraction of imaging phenotypes. Dr. Christos Davatzikos group from University of Pennsylvanian analyzed the baseline MRI T1 scans of ADNI1 participants and generated the 145 ROIs spanning the entire brain by using the Multi-atlas region Segmentation (MUSE) framework ${ }^{43}$. In this framework, multiple atlases with semi-automatically extracted ground-truth ROI labels were first warped individually to the target image using non-linear registration methods ${ }^{44,45}$. To fuse the ensemble into a final segmentation, they adopted a spatial adaptive weighted voting strategy, in which a local similarity term was used for ranking and weighting ground truth labels from different atlases and an image intensity based term was used for modulating the segmentations at the boundaries of the ROIs according to the intensity profile of the subject image ${ }^{43}$. In validation experiments, the multi-atlas approach was showed to achieve significantly higher accuracy in comparison to single-atlas based segmentation ${ }^{43}$. In this study, we downloaded the volume measures of ROIs from ADNI.

ROI-wise genome-wide association analysis in ADNI1. Autosomal chromosome SNP associations for volumes from 145 ROIs spanning whole brain were assessed by linear regression under the assumption of an additive genetic model. All models were adjusted for age, gender, education, handedness and 3 principal components to control population stratification. The genomic control for 145 GWASs ranged between 0.98 to 1.02 .

Genetic correlation network analysis of brain ROls in ADNI1. In multivariate quantitative genetics, a genetic correlation $\left(\boldsymbol{r}_{g}\right)$ is the proportion of variance that two traits share due to additive genetic effects, which estimates the degree of pleiotropy or causal overlap ${ }^{12,14,46}$. The cross phenotype association analysis (CPASSOC) is a method proposed to integrate association evidence of multiple traits from multiple GWAS and detect cross-phenotype associations ${ }^{13}$. Thus, CPASSOC analysis of genetic correlated AD-related brain imaging traits could improve power to identify genetic variants associated with multiple $\mathrm{AD}$-imaging traits. To identify groups of highly genetic correlated ROIs, we used the estimated pairwise ROI genetic correlations to define the brain genetic correlation network. In this network, nodes are brain ROIs while edges are estimated genetic correlations between ROIs. To extract modules from this network, we adopted a weighted gene co-expression network analysis (WGCNA) framework and used the method of topological overlap matrix (TOM) elements in hierarchical clustering to identify modular structures ${ }^{47}$. A flowchart for constructing a ROI genetic correlation network, extracting network modules and identifying genetic variants associated with modules using CPASSOC is presented in Supplementary Fig. 1a.

Pairwise ROI genetic correlations were estimated by the technique of cross-trait LD score regression method ${ }^{14}$ using the GWAS summary statistics of ROIs. For 10,400 pairs among 145 ROIs, genetic correlations were not correctly estimated for 3,255 pairs because the estimated values were either "NA", above 1 or below -1 , which might be driven by the small sample size, and these pairs were then filtered out. However, this filter may reduce power to identify variants associated with ROIs. The pairwise genetic correlations are presented in Supplementary Fig. 1 and we observed high genetic correlations among the ROIs. 
We used the ROI genetic correlation matrix and power adjacency function ${ }^{47}$ to generate network adjacent matrix:

$$
a_{i j}=\left|r_{g i j}\right|^{\beta}
$$

while $r_{g i j}$ is the genetic correlation between nodes ROI $i$ and ROI $j$, and $a_{i j}$ is the connection strength between two nodes.

To choose the parameter $\beta$ and genetic correlation $P$-value threshold, we used the scale-free network model to construct an image network. The scale-free network assumes that most nodes in a network are sparsely connected with the exception of a few hub nodes that are densely connected with other nodes ${ }^{48}$. In the scale-free network models, more connections are likely to occur for those hub nodes with already-high connectivity, which meet biological criteria ${ }^{47,48}$. We used the power law $p(k) \sim k^{-\gamma}$ to estimate the scale-free property, where $k$ is the connectivity for each node and equals the number of its direct connections to other node. To generate the network, we assessed different power adjacency function parameter $\beta=2,4,6$ and 8 and filtered the genetic correlation with different genetic correlation $\left(r_{g}\right) P$-value thresholds of $0.5,0.3,0.2$ and 0.1 . For each $P$-value threshold, if the estimated genetic correlation $P$-value was larger than that, we set the genetic correlation to be 0 . Using the four thresholds, we generated different networks for $\beta=2,4,6$ and 8 and accessed their corresponding scale-free topology using linear regression model fitting index $R^{2}$ between $\log _{10}(p(k))$ and $\log _{10}(k)$ for all nodes. We observed that a $P$-value threshold of 0.2 with $\beta=6$ corresponded a network with the scale-free topology and had the largest $R^{2}$ of 0.61 . The histogram of connectivity $k$ and scale-free topology plots for networks with $\beta=6$ and different P-value threshold were showed in Supplementary Fig. 2. Thus, we used the network adjacent matrix generated under this criterion for further analysis. In this network, 40 out of 145 ROIs had $k$ equal to 0 and 105 ROIs were carried out in module identification analysis.

We adopted the methods introduced by WGCNA framework ${ }^{47}$ to identify network modules. The adjacent matrix was transformed into a topological overlap matrix (TOM) with element defined as

$$
w_{i j}=\frac{l_{i j}+a_{i j}}{\min \left\{k_{i}, k_{j}\right\}+1-a_{i j}}
$$

where $l_{i j}=\sum_{u} a_{i j} a_{u j}$ and $k_{i}=\sum_{u} a_{i u}$ is the node connectivity.

TOM based dissimilarity measure was generated by

$$
d_{i j}^{w}=1-w_{i j}
$$

This dissimilarity matrix was used as the input for average linkage hierarchical clustering. The hierarchical clustering grouped the closet ROIs and formed the branches to identify module. For the genetic correlation network, we identified 16 modules spanning the whole brain with the largest module containing 17 ROIs and the smallest containing 3 ROIs (Supplementary Fig. 2).

CPASSOC analysis within modules. We applied the CPASSOC package developed by Zhu et al..$^{13}$ to combine association evidence of ROIs within each module. CPASSOC can integrate association evidence from summary statistics of multiple traits and improves power when variant is associated with at least one trait. CPASSOC provides two statistics, $\mathrm{S}_{\mathrm{Hom}}$ and $S_{\mathrm{Het}} . S_{\mathrm{Hom}}$ is similar to the fixed effect meta-analysis method ${ }^{49}$ but accounting for the correlation of summary statistics among cohorts induced by potential overlapped or related samples. In brief, assuming we have summary statistical results of GWAS from $J$ cohorts with $K$ phenotypic traits. In each cohort, single SNP-trait association was analyzed for each trait separately. Let $T_{j k}$ be a summary statistic for a SNP, $j^{\text {th }}$ cohort and $k^{\text {th }}$ trait. Let $\mathbf{T}=\left(T_{11}, \cdots, T_{J 1}, \cdots, T_{1 K}, \cdots, T_{J K}\right)^{T}$ represents a vector of test statistics for testing the association of a SNP with $K$ traits. Let

$\boldsymbol{\beta}=\left(\beta_{11, \ldots}, \beta_{J 1, \ldots}, \beta_{1 K, \ldots,} \beta_{J K}\right)^{T}$ be the effect sizes of the SNP. The null hypothesis is $\mathrm{H}_{0}: \boldsymbol{\beta}=\mathbf{0}$ and the alternative hypothesis $\mathrm{H}_{1}$ is that at least one of the elements of $\boldsymbol{\beta}$ is not equal to zero. We used a Wald test statistic $T_{j k}=\widehat{\beta}_{j k} / \widehat{s}_{j k}$, where $\hat{\beta}_{j k}$ and $\hat{s}_{j k}$ are the estimated coefficient and corresponding standard error for the $k^{\text {th }}$ trait in the $j^{\text {th }}$ cohort. It is reasonable to assume that $\mathbf{T}$ follows a multivariate normal distribution with mean $\mathbf{0}$ and correlation matrix $R$ under the null hypothesis. When the effect is homogeneous, the most powerful test statistic $S_{\mathrm{Hom}}$ is defined as

$$
S_{\mathrm{Hom}}=\frac{e^{T}(\mathrm{RW})^{-1} T\left(e^{T}(\mathrm{RW})^{-1} T\right)^{T}}{e^{T}(\mathrm{WRW})^{-1} e}
$$

which follows a $\chi^{2}$ distribution with one degree of freedom, where $\mathbf{e}^{\mathrm{T}}=(1, \ldots, 1)$ has length $J \times K$ and $W$ is a diagonal matrix of weights for the individual test statistics. We used the sample sizes for the weights, $w_{j k}=\sqrt{n_{j}}, n_{j}$ is sample size of the $j^{\text {th }}$ cohort.

To further allow for different effect directions of a variant for different traits in different cohorts, we define $S_{\mathrm{Het}}$. We first let

$$
S(\tau)=\frac{\left.e^{T}(R(\tau) W(\tau))^{-1} T(\tau)(R(\tau) W(\tau))^{-1} T(\tau)\right)^{T}}{e^{T} W(\tau)^{-1} R(\tau)^{-1} W(\tau)^{-1} e}
$$

Where $\mathbf{T}(\boldsymbol{\tau})$ is the sub-vector of $\mathbf{T}$ satisfying $\left|T_{j k}\right|>\tau$ for a given $\tau>0, R(\tau)$ is a sub-matrix of $R$ representing the correlation matrix, and $W(\tau)$ be the diagonal submatrix of $W$, corresponding to $\mathbf{T}(\boldsymbol{\tau})$. The test statistic is then

$$
S_{H e t}=\max _{\tau>0} S(\tau)
$$

The asymptotic distribution of $S_{\mathrm{Het}}$ does not follow a standard distribution but can be evaluated using simulation. $S_{\mathrm{Het}}$ is an extension of $S_{\mathrm{Hom}}$ but power can be improved when the genetic effect sizes vary for different traits. The distribution of $S_{\mathrm{Het}}$ under the null hypothesis can be obtained through simulations or approximated by an estimated beta distribution.

We applied both $S_{\mathrm{Hom}}$ and $S_{\mathrm{Het}}$ to combine summary statistics for ROIs within each module. The CPASSOC analysis of multiple genetic correlated traits in identified module would allow us to identify variants that are likely to be missed by conventional GWAS of single trait and reduce the multiple comparison burden in the genetic analysis of hundreds of neuroimaging traits. Finally, we identified 15 loci with CPASSOC test $P$-value less than $1 \times 10^{-7}$ in nine modules (Supplementary Table 1). Importantly, three previously reported AD-associated SNPs, rs429358, rs2075650 and rs439401 and the FAM222A SNP rs117028417 were exclusively found in one module, which were green colored in Supplementary Fig. 1M and Supplementary Table 1. The Manhattan plots and Q-Q plots of CPASSOC analysis and single ROI GWAS for this module were showed in Fig. 1 and Supplementary Fig. 2.

Genetic analysis of AV-45 PET imaging. ${ }^{18} \mathrm{~F}$-Florbetapir (AV-45) PET imaging was performed at baseline and 2-year follow-up for participants enrolled in the ADNI GO and two phases ${ }^{22}$. UC Berkeley extracted weighted AV-45 standardized uptake value ratio (SUVR) means for four main cortical regions: frontal, anterior, and posterior cingulate, lateral parietal and lateral temporal regions (version 2019.4.12) for ADNI-GO2 participants. They also calculated composite SUVR for cortical which is weighted SUVR mean in frontal, cingulate, parietal and temporal regions. These data can be downloaded from the ADNI database. We used the SUVR mean of composite region including whole cerebellum, pons/brainstem and eroded white matter as reference. Mean AV-45 SUVR of frontal, cingulate, lateral parietal, lateral temporal and composite cortical relative to the reference were calculated. The annual percent change in SUVR means at 2-year follow-up compared to baseline was used as the main quantitative phenotype for genetic analysis. The annual percent changes in AV-45 SUVR for all five brain regions were approximately normally distributed (Supplementary Fig. 20). We collected 369 individuals with both SUVR measures for baseline and 2-year follow-up and whole-genome sequencing data. The samples included 120 healthy people, 26 people with $\mathrm{AD}, 64$ people with late mild cognitive impairment (LMCI) and 159 people with early mild cognitive impairment (EMCI) diagnosed at baseline. The samples characteristics and demographics for samples are shown in Supplementary Table 11.

WGS data from 817 ADNI participants were downloaded from the ADNI dataset. WGS was performed using blood-derived genomic DNA samples and sequenced on the Illumina HiSeq2000 using paired-end read chemistry and read lengths of $100 \mathrm{bp}$ at $30-40 \mathrm{X}$ coverage $\mathrm{5}^{50}$. As previously described using Broad GATK and BWA-mem, reads were mapped and aligned to the human genome (build 37), then variants were called ${ }^{50,51}$.

For single SNP association test, association test of SNP rs117028417 with phenotypes were performed using linear regression under an additive genetic model in PLINK. Baseline age and gender were included as covariates. For genebased association test, we extracted 8 and 6 functional coding variants defined as missense, in frame deletion/insertion, stop gained/lost, start gained/lost, splice acceptor/donor, or initiator/start codon for FAM222A and TRPV4 respectively. All of those variants are rare with minor allele frequency (MAF) $<0.01$ in ADNI samples. Gene-based association tests were performed using burden and SKAT ${ }^{52}$, adjusting age and sex as covariates.

Genetic analysis of CSF A $\boldsymbol{\beta}$ and Tau. Collection and processing of ADNI CSF samples was described in the ADNI procedures manual (http://www.adni-info.org/). We downloaded UPENNBIOMKs dataset.csv file from ADNI website. We collected 617 individuals with both CSF $A \beta_{42}$, tTau and pTau at baseline level and WGS data. For baseline data, since raw CSF biomarkers were skewed or bimodal skewed distributed, rank normal transformations were conducted for each biomarker separately (Supplementary Fig. 21a-f). To conduct CSF biomarkers longitudinal change genetic association, we collected 274 individuals with both baseline and 24-month follow-up CSF biomarkers and WGS data. The CSF biomarkers raw data at baseline and 2-year follow-up in 218 individuals were used to calculate annual changes in $A \beta_{42}$, tTau and pTau separately. The annual changes of three CSF biomarkers were approximately normally distributed (Supplementary Fig. 21g-i). The samples characteristics and demographics for CSF biomarker traits association analysis are shown in Supplementary Tables 12, 13.

Association test of SNP rs117028417 with phenotypes were performed using linear regression under an additive genetic model in PLINK. Baseline age and sex were included as covariates. We extracted 8 and 15 coding variants defined as missense, in frame deletion/insertion, stop gained/lost, start gained/lost, splice acceptor/donor, or initiator/start codon for FAM222A and TRPV4 respectively (Supplementary Table 4). All of those variants are rare with minor allele frequency 
$(\mathrm{MAF})<0.01$ in ADNI samples. Gene-based association tests were performed using burden and SKAT $^{52}$, adjusting age and sex as covariates.

Analysis of FAM222A mRNA in AD. The development of the Mount Sinai Brain Bank (MSBB) cohort was described in the previous studies ${ }^{35,53}$. MSBB is a large $\mathrm{AD}$ cohort and now holds over 2,040 well-characterized human brains ${ }^{53}$. The datasets we used assessed a total of 125 human brains which was assembled after applying stringent inclusion/exclusion criteria and represents the full spectrum of cognitive and neuropathological disease severity ${ }^{35}$. Detailed sample demographic information and description of the cognitive and neuropathological traits can be seen in previously published paper by Dr. Bin Zhang's lab ${ }^{35}$. We downloaded the normalized microarray data of MSBB Array Tissue Panel Study from the Synapse at http://www.synapse.org/\#!Synapse:syn3157699. The RNA samples from 19 brain regions isolated from $125 \mathrm{MSBB}$ specimens were collected and profiled on the Affymetrix $133 \mathrm{AB}$ and Affymetrix 133Plus2 platforms. RNA quality was assessed using a combination of a 260/280 ratio derived from resolution electrophoresis system (LabChipTM, Agilent Technologies, Palo Alto, CA, USA) and $3^{\prime}-5^{\prime}$ hybridization ratios for GAPDH probes ${ }^{35}$. Not all brain regions for all subjects were available for analysis. There was an approximately 60 samples (40 AD, 20 controls) per brain region available for analysis. The array probes were annotated according to the Ensemble version 72 (genome build GRCh37) using the R/Biomart library. The raw microarray data were quantile normalized with all probe sets on the arrays using $\mathrm{RMA}^{54}$ method implemented in the R/Bioconductor package affy (v1.44) with the default parameters. The data were then corrected for covariates including sex, postmortem interval (PMI), $\mathrm{pH}$ and race using a linear regression model. The FAM222A gene expression data was identified by probe set 226487_at. The processed FAM222A mRNA level means for groups of AD and control were compared using two-sided Welch t-test using R.

\section{Association analysis of FAM222A DNA methylation. We downloaded two} datasets, E-GEOD-45775 and E-GEOD-76105, with DNA methylation profiling from the European Bioinformatics Institute (EMBL-EBI) ArrayExpress website https://www.ebi.ac.uk/arrayexpress/. Samples of dataset E-GEOD-45775 included 5 controls, 5 AD Braak stage I-II and 5 AD Braak stage V-VI (Supplementary Table 14). The methylation values were adjusted and normalized using BeadStudio software v3.2 to obtain normalized beta and average Beta detect P-value. The array used the HumanMethylation27_270596_v.1.2 design and one methylation site cg01335367 was identified located on chr12:109734355-109734404 (GRCh38.p12), associated with FAM222A. We analyzed the association between methylation in $\operatorname{cg} 01335367$ with AD using logistic regression and adjusted for sex. We also performed one-way analysis of variance (ANOVA) to determine differences between methylation levels of control and different Alzheimer Braak stage groups. Study EGEOD-70615 investigated DNA methylation profiling in the superior temporal gyrus (STG). Samples included $34 \mathrm{AD}$ and 34 non-demented controls, which had 52 European, 8 Hispanic, 6 African, 1 Asian Americans and 1 unknown (Supplementary Table 15). The Beta values from the probes were quantile normalized using lumi package in R. We performed association analysis in 52 European Americans only. The association between methylation in those sites with AD were analyzed using logistic regression model adjusting age, gender and estimated cellular proportions (neuronal vs. glial).

Mice and human tissues. Mouse surgery and procedures were performed according to the NIH guidelines and were approved by the Institutional Animal Care and Use Committee (IACUC) at Case Western Reserve University. 5xFAD transgenic mice (B6.Cg-Tg(APPSwFlLon, PSEN1*M146L*L286V) 6799Vas/ Mmjax, JAX\#008730) were purchased from the Jackson Laboratory. The use of all human tissue samples was approved by the University Hospitals Institutional Review Board (IRB) for human investigation at University Hospitals Case Medical Center at Cleveland. Human brain tissues obtained postmortemly from University Hospitals of Cleveland were fixed, and $6-\mu \mathrm{m}$-thick consecutive sections were prepared. The information of fixed or frozen human tissues were listed in Supplementary Tables 16, 17.

\section{Immunocytochemistry, immunofluorescence and immunoblot. Immunocy-} tochemistry was performed by the peroxidase anti-peroxidase protocol. Taken briefly, paraffin embedded brain tissue sections were first deparaffinized in xylene and rehydration in graded ethanol and incubated in Tris Buffered Saline (TBS, $50 \mathrm{mM}$ Tris- $\mathrm{HCl}$ and $150 \mathrm{mM} \mathrm{NaCl}, \mathrm{pH}$ 7.6) for $10 \mathrm{~min}$ before antigen retrieval in 1X Immuno/DNA retriever with citrate (BioSB, Santa Barbara, CA) under pressure using BioSB's TintoRetriever pressure cooker. Sections were rinsed with distilled $\mathrm{H}_{2} \mathrm{O}$, and blocked with $10 \%$ normal goat serum (NGS) in TBS at room temperature (RT) for $30 \mathrm{~min}$. Tissue sections were further incubated with primary antibodies in TBS containing $1 \%$ NGS overnight at $4{ }^{\circ} \mathrm{C}$, and immunostained by the peroxidaseantiperoxidase based method. For double Immunofluorescence staining, paraffin embedded tissue sections were deparaffinized in xylene and re-hydrated in graded ethanol without $\mathrm{H}_{2} \mathrm{O}_{2}$ incubation as described above. The sections were incubated in phosphate buffered saline (PBS) at RT for 10 min followed by block with $10 \%$ NGS in PBS for $45 \mathrm{~min}$ at RT. The sections were incubated with primary antibodies in PBS containing $1 \%$ NGS overnight at $4{ }^{\circ} \mathrm{C}$. After being washed with $1 \%$ NGS in
PBS for $10 \mathrm{~min}$, the sections were incubated in 10\% NGS for $10 \mathrm{~min}$ and followed by three quick washes with $1 \%$ NGS in PBS. Then, the sections were incubated with Alexa Fluor 488 or 568 dye labeled secondary antibodies (1:300, Invitrogen, Carlsbad, CA) for $2 \mathrm{~h}$ at RT in dark, washed three times with PBS, stained with DAPI, washed again with PBS for three times, and finally mounted with Fluoromount-G mounting medium (Southern Biotech, Birmingham, AL). For thioflavin-S staining, slides were incubated with $1 \%$ thioflavin-S (Santa Cruz Biotechnology, Dallas, TX) for $8 \mathrm{~min}$, washed 2 times with $80 \%$ ethanol, and 1 time with $95 \%$ ethanol and PBS, then stained with DAPI. For immunoblot, human or mice tissue samples were all lysed with TBS plus $1 \mathrm{mM}$ phenylmethylsulfonyl fluoride (PMSF) (Millipore, Burlington, MA), protease inhibitor cocktail (Sigma Aldrich, St. Louis, MO) and phosphatase inhibitor cocktail (Sigma Aldrich, St. Louis, MO). Equal amounts of total protein extract were resolved by SDS-PAGE and transferred to Immobilon-P (Millipore, Burlington, MA). Following blocking with $10 \%$ nonfat dry milk, primary and secondary antibodies were applied and the blots developed with Immobilon Western Chemiluminescent HRP Substrate (Millipore, Burlington, MA). Images were taken by ChemiDoc Touch Imager (Biorad, Hercules, CA). Primary antibodies used in this study are listed in Supplementary Table 18. The dilution of antibodies used for IF or IHC. 4G8 (BioLegend, SIG-39220; IF, 1:1000), 6E10 (BioLegend, 803001; IF and IHC, 1:1000), 82E1 (IBL, 10323; IF, 1:1000), Aggregatin (Abcam, ab122626; IF/IHC, 1:100), Aggregatin (LifeSpan BioSciences, LS-C170630; IHC, 1:1000), Aggregatin (Aviva Systems Biology, ARP69038_P050; IHC, 1:1000), Flag (Sigma Aldrich, F1804; IF/IHC 1:1000), Flag (Thermo Fisher, PA1-984B; IHC, 1:200), Flag (Cell Signaling Technology, 2368; IHC, 1:200), Flag-HRP (Proteintech, HRP-66008; IHC, 1:1000), GFP (Abcam, ab32146; IHC, 1:500), Myc (Thermo Fisher, MA1-21316; IHC, 1:1000), Myc (Cell Signaling Technology, 2276; IHC, 1:500), and Nu4 (Klein lab, IF/IHC, 1:2000). All uncropped and unprocessed blots are provided in the Source Data file (Source Data for Statistics and Blots).

Expression vectors and recombinant proteins. pcDNA3.1(+) (Invitrogen, Carlsbad, CA) plasmid was modified to express recombinant proteins to express recombinant proteins containing a $4 x$ Flag-Twin-Strep-tag at their $\mathrm{N}$-terminal. The cDNA of full length or truncated human Aggregatin were inserted into the modified pcDNA3.1(+) plasmid. All primers and cDNA constructs used in this study are listed in Supplementary Data 1 and Supplementary Table 19. Eight micrograms plasmid was used to transfect one $10 \mathrm{~cm}$ dish of Lenti-293T cells with TransIT $^{\circledast}-293$ Transfection Reagent (Mirus, Madison, WI). Cells were collected at $24 \mathrm{~h}$ after transfection and lysed by lysis buffer $(100 \mathrm{mM}$ Tris- $\mathrm{HCl}, 150 \mathrm{mM} \mathrm{NaCl}$, $1 \mathrm{mM}$ EDTA and 1\% NP40, pH 8.0) containing $1 \mathrm{mM}$ PMSF (Millipore, Burlington, MA), protease inhibitor cocktail (Sigma Aldrich, St. Louis, MO) and phosphatase inhibitor cocktail (Sigma Aldrich, St. Louis, MO). The lysate was centrifuged at $14,000 \mathrm{~g}$ for $15 \mathrm{~min}$ at $4{ }^{\circ} \mathrm{C}$. Supernatant was incubated with MagStrep type 3 XT beads (IBA Lifesciences, Goettingen, Germany) overnight at $4{ }^{\circ} \mathrm{C}$ Beads were washed three times with lysis buffer, and eluted with BXT buffer (IBA Lifesciences, Goettingen, Germany) overnight at $4{ }^{\circ} \mathrm{C}$. At last, the eluted recombinant proteins were subjected to dialysis using $10 \mathrm{kD}$ Slide-A-Lyzer ${ }^{\mathrm{TN}}$ Dialysis Cassettes (Thermo Fisher Scientific, Waltham, MA), concentration with $10 \mathrm{kD}$ Spin Column (Abcam, Cambridge, MA) and purification by size-exclusion chromatography.

Stereotaxic injection and ICV infusion. Mice surgery were performed according to the NIH guidelines and were approved by the Institutional Animal Care and Use Committee (IACUC) at Case Western Reserve University. All AAVs with $10^{13}$ genome copies per $\mathrm{mL}(\mathrm{GC}$ per $\mathrm{mL}$ ) were obtained from Vigene Biosciences (Jinan, China). For stereotaxic injection, mice were anesthetized with isoflurane and immobilized using the stereotactic frame equipped with a heating blanket to maintain body temperature throughout the procedure. After hair removal and the cleaning of the shaved area with betadine and alcohol, mice were injected with bupivacaine/lidocaine and a small incision was made to expose the skull surface. Two small holes were drilled in the skull (relative to bregma: anteroposterior $-2.1 \mathrm{~mm}$, medial lateral $\pm 2 \mathrm{~mm}$; Note that \pm is the plus-minus sign throughout this study) followed by injection of $2 \mu \mathrm{l}$ AAVs using Hamilton syringes into the hippocampal CA1 at dorsal ventral $-1.45 \mathrm{~mm}$. Injection speed was pump controlled at $0.2 \mu \mathrm{l}$ per min. The needle was left in place for $5 \mathrm{~min}$ before it was slowly withdrawn. For ICV infusion, the mini-osmotic pump (Model 1004, Alzet, Cupertino, CA; flow rate of $0.11 \mu \mathrm{l}$ per hour, 28 days) and brain infusion cannula attached with $2.5-3 \mathrm{~cm}$ catheter tubes (Brain infusion kit 3, Alzet, Cupertino, CA) were filled with recombinant protein in artificial cerebrospinal fluid (aCSF), followed by pump incubation in aCSF at $37^{\circ} \mathrm{C}$ for $48 \mathrm{~h}$ according to the manufacturer's instructions For implant surgery, a hole was drilled in the skull (relative to bregma: anteroposterior $-0.5 \mathrm{~mm}$, medial lateral $0.75 \mathrm{~mm}$ ). The cannula was positioned on the skull with the needle plug $2.5 \mathrm{~mm}$ into the ventricle. The cannula was fixed and secured by cyanoacrylate glue.

Behavioral tests. Mice behavioral tests were also performed according to the $\mathrm{NIH}$ guidelines and were approved by the Institutional Animal Care and Use Committee (IACUC) at Case Western Reserve University. The Barnes maze consisted of a white acrylic circular disk $92 \mathrm{~cm}$ in diameter with 20 equally spaced holes $(5 \mathrm{~cm}$ in 
diameter) located $2 \mathrm{~cm}$ from the edge of the disk. The maze was illuminated by two $60 \mathrm{~W}$ lamps to provide an aversive, bright disk surface. An acrylic escape box $(7 \times 7 \times$ $5 \mathrm{~cm}$ ) could be fitted under any of the holes in the maze. The maze was raised $30 \mathrm{~cm}$ from the floor and rested on a pedestal that enabled it to be rotated $360^{\circ}$ on a horizontal plane. An acrylic start bin with $15 \mathrm{~cm}$ diameter and $15 \mathrm{~cm}$ height was used. Trials were recorded using a webcam and analyzed by video tracking software (EthoVision XT, Noldus, Leesburg, VA). Each trial began with the start bin positioned in the center of the maze with the mouse placed inside. The mouse remained in the start bin for $30 \mathrm{~s}$, providing a standard starting context for each trial and ensuring that initial orientation of the mouse in the maze varied randomly from trial to trial. Each mouse was allowed to explore the maze freely for $2 \mathrm{~min}$. After the mouse entered the escape hole, the mouse was left in the escape box for $90 \mathrm{~s}$ before being returned to its home cage. If the mouse did not enter the escape box within $120 \mathrm{~s}$, it was gently picked up by the experimenter and placed over the target hole and allowed to enter the escape box. After each trial, the maze and escape box were cleaned carefully with a $10 \%$ alcohol solution to dissipate odor cues and provide a standard olfactory context. Five training sessions consisting of two trials each were run on subsequent days and escape latencies were measured. For Y maze test, mice were placed in a Plexiglas Y maze (with arms $60 \mathrm{~cm}$ in length) and allowed to explore the maze freely for $10 \mathrm{~min}$. When put in the Y maze, the mice were recorded using the ANY-maze tracking system, and the time and frequency in the spontaneous alteration ratio were counted automatically. All tests were performed at the Case Behavior Core, with the investigator blinded to mouse genotype.

Plaque isolation. Amyloid plaque cores were isolated as previously described ${ }^{55}$. Briefly, whole mouse brain or human brain gray matters were homogenized, boiled

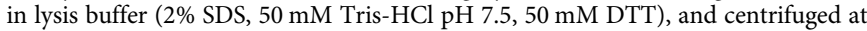
$100,000 \mathrm{~g}$ for $1 \mathrm{~h}$ at $10^{\circ} \mathrm{C}$. The pellet was solubilized in fraction buffer $(1 \%$ SDS, 50 $\mathrm{mM}$ Tris- $\mathrm{HCl} \mathrm{pH} \mathrm{7.5,50} \mathrm{mM} \mathrm{DTT)} \mathrm{and} \mathrm{centrifuged} \mathrm{at} 100,000 \mathrm{~g}$ for $1 \mathrm{~h}$ at $10^{\circ} \mathrm{C}$. The pellet was further suspended in fraction buffer and loaded on top of a discontinuous sucrose gradient $(1.0,1.2,1.4$ and $2.0 \mathrm{M}$ sucrose in $50 \mathrm{mM}$ Tris $\mathrm{pH} 7.5$ containing $1 \%$ SDS), centrifuged at $220,000 \mathrm{~g}$ for $20 \mathrm{~h}$ at $10^{\circ} \mathrm{C}$ and fractionated into sixteen fractions $(300 \mu \mathrm{l}$ per fraction). Plaque-core-enriched fraction \#13 were further diluted in fraction buffer and centrifuged at $220,000 \mathrm{~g}$ for $2 \mathrm{~h}$ at $10^{\circ} \mathrm{C}$. The resulting pellet was dissolved in $70 \%$ formic acid and subsequently dried using a SpeedVac system. Solubilized proteins were further resuspended in 1X SDS sample buffer with $8 \mathrm{M}$ Urea.

A $\beta$ preparation, pull-down, and co-sedimentation assay. Synthetic human $\mathrm{A} \beta_{1-42}$ and $\mathrm{A} \beta_{1-40}$ peptides (GL Biochem, Shanghai) were dissolved in hydroxylfluro-isopro-panol (HFIP) and subsequently dried using a SpeedVac system. Both $A \beta_{1-42}$ and $A \beta_{1-40}$ monomers were prepared by dissolving the lyophilized $A \beta$ in dimethyl sulfoxide (DMSO) at $5 \mathrm{mM}$, sonicated for $10 \mathrm{~min}$ and diluted in PBS buffer ( $\left.\mathrm{NaCl} 137 \mathrm{mM}, \mathrm{KCl} 2.7 \mathrm{mM}, \mathrm{Na}_{2} \mathrm{HPO}_{4} 10 \mathrm{mM}, \mathrm{KH}_{2} \mathrm{PO}_{4} 1.8 \mathrm{mM}, \mathrm{pH} 7.4\right)$ to different concentrations. $\mathrm{A} \beta_{1-42}$ oligomers were prepared in DMSO/PBS and oligomerized by incubation at $4{ }^{\circ} \mathrm{C}$ for 24 or $48 \mathrm{~h}$. Monomeric or oligomer $A \beta_{1-40}$ $(100 \mu \mathrm{M})$ and $A \beta_{1-42}$ solutions $(50 \mu \mathrm{M})$ supplemented with or without rAggregatin bound Strevdin-avdin beads were incubated in IP buffer $(\mathrm{NaCl} 300 \mathrm{mM}, \mathrm{KCl}$ $2.7 \mathrm{mM}, \mathrm{Na} 2 \mathrm{HPO} 410 \mathrm{mM}, \mathrm{KH} 2 \mathrm{PO} 41.8 \mathrm{mM}, \mathrm{pH} 7.4)$ at RT with shaking for $2 \mathrm{~h}$. After 4 times wash with IP buffer, beads were eluted by 1XSDS sample buffer ( 32.9 $\mathrm{mM}$ Tris- $\mathrm{HCl} \mathrm{pH} 6.8,13 \%$ Glycerol, $1 \%$ SDS and $0.005 \%$ bromophenol blue) and analyzed by $10-20 \%$ SDS/Tricine protein gels (Invitrogen, Carlsbad, CA). For $\mathrm{A} \beta_{1-42}$ oligomer formation and co-sedimentation assay, HFIP dissolved synthetic $\mathrm{A} \beta_{1-42}$ peptides were solubilized in $30 \mathrm{mM} \mathrm{NaOH}$ to a final concentration of $100 \mu \mathrm{M}$, diluted to $2.5 \mu \mathrm{M}$ in PBS and incubated with and without $30 \mathrm{nM} \mathrm{rAg-}$ gregatin at $37^{\circ} \mathrm{C}$ for different time points. After 10 -minute centrifuge at $14,000 \mathrm{~g}$, pellets and supernatants were collected and analyzed by $10-20 \%$ SDS/Tricine protein gels (Invitrogen, Carlsbad, CA).

Dynamic light scattering. Dynamic light scattering (DLS) experiments were carried out with DynaPro ${ }^{\text {Tx }}$ instrument from Wyatt technology with a wavelength of $633 \mathrm{~nm}$ and a scattering angle of $173^{\circ}$. The measurements of Aggregatin or Aggregatin $\Delta 61-80$ at $100 \mathrm{nM}$ were performed at $25^{\circ} \mathrm{C}$ after 2 min equilibration with correlation times defined on $10 \mathrm{~s}$ per run with 30 runs for each measurement. The results were plotted as intensity of distribution (\%) of particles versus hydrodynamic radius $(\mathrm{nm})$.

Circular dichroisms. The spectra were recorded over a wavelength range of 260-190 nm with standard sensitivity at the $50 \mathrm{~nm}$ per min scan speed with $1-\mathrm{nm}$ resolution and 1-s time constant at room temperature using a spectropolarimeter (Jasco J-815). All the proteins were dissolved in phosphate buffer (pH8.0). The final concentration of all samples was $1 \mu \mathrm{M}$. The secondary structure content was calculated from the Circular dichroisms (CD) spectra using the online software K2D3.

Surface plasmon resonance. Surface plasmon resonance (SPR) was determined using BIAcore3000 (GE Healthcare Life Sciences, Pittsburgh, PA). rAggregatin ( $0.1 \mathrm{mg}$ per $\mathrm{ml}$ ) was immobilized on the CM5 sensor surface (GE Healthcare Life Sciences, Pittsburgh, PA) in $10 \mathrm{mM}$ acetate buffer $(\mathrm{pH}=4.5)$. Running buffer was $1 \%$ DMSO in PBS-P buffer $(0.02 \mathrm{M}$ phosphate, $2.7 \mathrm{mM} \mathrm{KCl}, 137 \mathrm{mM} \mathrm{NaCl}$ and
$0.05 \%$ Tween 20). Binding of a dilution series comprising $\mathrm{A} \beta_{1-42}$ monomers to rAggregatin was analyzed and fitted to the 1:1 binding model using BIAevaluation software (GE Healthcare Life Sciences, Pittsburgh, PA).

Solid phase binding assay. rAggregatin was coated onto Nunc MaxiSorp 96-well plates (Thermo Fisher Scientific, Waltham, MA) at $0.1 \mu \mathrm{g}$ per well in PBS at $4{ }^{\circ} \mathrm{C}$ overnight. After blocking in $1 \%$ BSA in PBS for $2 \mathrm{~h}$ at RT, $\mathrm{A} \beta_{1-42}$ at $6.25,12.5,25$, 50,100 , or $200 \mathrm{nM}$ or $\mathrm{A} \beta_{1-40}$ at $0.5,1,2,4$, or 8 , or $16 \mu \mathrm{M}$ monomers were added to the plates at $4{ }^{\circ} \mathrm{C}$ overnight. Plates were washed with PBS 4 times and incubated with $6 \mathrm{E} 10$ antibody at $4{ }^{\circ} \mathrm{C}$ overnight, followed by 4 times PBS wash and development in TMB solution (Thermo Fisher Scientific, Waltham, MA). The reaction was stopped by sulfuric acid and assessed using a Synergy $\mathrm{H} 1$ microplate reader (BioTek, Winooski, VT). Likewise, $0.2 \mu \mathrm{g} A \beta_{1-42}$ or $\mathrm{A} \beta_{1-40}$ monomers were immobilized on plates and incubated with $3.125,6.25,12.5,25,50$, or $100 \mathrm{nM}$ rAggregatin. Bound rAggregatin were detected by an anti-Flag antibody and developed in TMB solution as described above.

ThT fluorescence assay. HFIP treated $A \beta_{1-40}$ or $A \beta_{1-42}$ peptides were solubilized in $30 \mathrm{mM} \mathrm{NaOH}$ to a final concentration of $400 \mu \mathrm{M}$, sonicated for $5 \mathrm{~min}$ in a water bath and stored at $-80^{\circ} \mathrm{C}$ until further use. To monitor $\mathrm{A} \beta_{1-40}$ and $\mathrm{A} \beta_{1-42}$ fibrillization, a ThT assay was performed according previous studies ${ }^{56,57}$. Briefly, a stock solution of A $\beta$ was diluted to in PBS with $20 \mu \mathrm{M}$ ThT. Then rAggregatin were added at desired concentrations to the final volume of $100 \mu \mathrm{l}$. All samples were transferred to a black 96-well nonbinding Surface microplate with clear bottom (Corning, Corning, NY), and sealed with a polyester-based sealing film (Corning, Corning, NY). Samples were incubated at $37^{\circ} \mathrm{C}$ with stirring. Real-time ThT fluorescence was measured every $5 \mathrm{~min}$ for at least $12 \mathrm{~h}$ at the excitation and emission wavelengths of $446 \mathrm{~nm}$ and $482 \mathrm{~nm}$ respectively by a Synergy $\mathrm{H} 1$ microplate reader (BioTek, Winooski, VT).

$\mathbf{A} \boldsymbol{\beta}_{1-42}$ aggregates stained by Thio-S. To evaluate $A \beta$ aggregates formed in vitro, rAggregatin $(30 \mathrm{nM})$ and $2.5 \mu \mathrm{M} \mathrm{A} \beta$ in PBS were incubated at $37^{\circ} \mathrm{C}$ for 4 weeks. $20 \mu \mathrm{l}$ of protein solution were applied to the glass slides and completely air dry for $30 \mathrm{~min}$. After washing with PBS, the samples were stained by $1 \%$ Thio-S for $10 \mathrm{~min}$. The 3D confocal images were analyzed by using Imaris (Bitplane, Concord, MA) and the structure surface were extracted by using the SURFACE tools following the manufacturer's instructions.

Negative electric microscopy. HFIP dissolved synthetic $\mathrm{A} \beta_{1-42}$ peptides were solubilized in $30 \mathrm{mM} \mathrm{NaOH}$ to a final concentration of $100 \mu \mathrm{M}$. Then diluted to $2.5 \mu \mathrm{M}$ in PBS and incubated with and without $30 \mathrm{nM}$ rAggregatin at $37^{\circ} \mathrm{C}$. Immediately following the indicated incubation time, $20 \mu \mathrm{l}$ of protein solution were applied to the support surface of the grids, which were autoclaved by UV irradiation overnight. The grids were washed with $20 \mu \mathrm{l}$ droplets of water 4 times, followed by a $20 \mu \mathrm{L}$ droplet of uranyl acetate solution, then examined in an FEI Tecnai Spirit (T12) with a Gatan US4000 4kx4k CCD.

Total A $\boldsymbol{\beta}$ measurement by ELISA. Brains were homogenized in TBS Buffer (50 mM Tris- $\mathrm{HCl}$ and $150 \mathrm{mM} \mathrm{NaCl}, \mathrm{pH}$ 7.6) containing $1 \mathrm{mM}$ PMSF (Millipore, Burlington, MA), protease inhibitor cocktail (Sigma Aldrich, St. Louis, MO) and phosphatase inhibitor cocktail (Sigma Aldrich, St. Louis, MO). Total protein concentrations were determined using the BCA kit (Thermo Fisher Scientific, Waltham, MA). ELISA of total A $\beta$ was carried out in 96-well high-binding microtiter plates. Monoclonal antibody $6 \mathrm{E} 10$ raised against residues $\mathrm{A} \beta 1-16$ was used as a capture antibody (diluted in PBS pH 7.4) and incubated over night at $4^{\circ}$ Cin a humid chamber. After removal of the capture antibody, the plate surface was blocking with $1 \% \mathrm{BSA}$ for $1.5 \mathrm{~h}$. After washing with PBS, $0.5 \mu \mathrm{g}$ total protein were added and incubated at $4{ }^{\circ}$ Covernight. Monoclonal antibody MOAB- 2 coupled to horseradish peroxidase diluted in PBS were used as secondary antibodies and again incubated over night at $4^{\circ} \mathrm{C}$. After three times washing with PBS, $100 \mu \mathrm{l}$ of TMB ELISA peroxidase substrate (Thermo Fisher Scientific, Waltham, MA) was added and incubated for 1-10 min at RT in darkness. The reaction was stopped with 100 $\mu \mathrm{l} 2 \mathrm{M} \mathrm{H}_{2} \mathrm{SO}_{4}$ and absorbance was measured in a microplate reader at $450 \mathrm{~nm}$. For generation of standard curves, synthetic $\mathrm{A} \beta 1-42$ peptides freshly dissolved in DMSO from 1 ng per $\mu \mathrm{L}$ to 10 pg per $\mu \mathrm{L}$.

Isolation of exosomes. Lenti-293T cells were transfected with empty vector or pCDNA-4xFlag-Aggregatin using TransIT ${ }^{\circledR}-293$ Transfection Reagent (Mirus, Madison, WI). Twenty-four hours after transfection, cells were cultured in the DMEM medium supplemented with exosome-free FBS. Forty-eight hours later, the cell culture medium was collected and centrifuged at $300 \mathrm{~g}$ for $15 \mathrm{~min}$ to remove cells and debris. The supernatant was further filtered through a $0.22 \mu \mathrm{m}$ filter and centrifuged at $100,000 \mathrm{~g}$ for $2 \mathrm{~h}$ at $4^{\circ} \mathrm{C}$. The pellets enriched with exosomes were resuspended in the lysis buffer $(100 \mathrm{mM}$ Tris- $\mathrm{HCl}, 150 \mathrm{mM} \mathrm{NaCl}, 1 \mathrm{mM}$ EDTA and $1 \%$ NP40, pH 8.0) containing 1 mM PMSF (Millipore, Burlington, MA), protease inhibitor cocktail (Sigma Aldrich, St. Louis, MO), and phosphatase inhibitor cocktail (Sigma Aldrich, St. Louis, MO) followed by immunoblot analysis. 
Confocal microscopy and image analysis. All fluorescence images were imaged on a Leica TCS SP8 gSTED confocal microscopy (Leica Microsystems, Buffalo Grove, IL) equipped with a motorized super Z galvo stage, two PMTs, three Hyd SP GaAsP detectors for gated imaging, and the AOBS system lasers including a 405 $\mathrm{nm}$, Argon (458, 476, 488, 496, $514 \mathrm{~nm}$ ), a tunable white light ( 470 to $670 \mathrm{~nm}$ ), and a $592 \mathrm{~nm}$ STED depletion laser. Series of confocal images with optical thickness of $300 \mathrm{~nm}$ were collected using the $\times 100$ oil objective. All 3D confocal images of plaque were reconstructed using Imaris (Bitplane, Concord, MA) after background subtraction. Quantification of Aggregatin foci in plaques and measurement of plaque load and size were performed with open-source image analysis programs WCIF ImageJ (developed by W. Rasband).

Statistical analysis. Statistical analysis was done with one-way analysis of variance (ANOVA) followed by Tukey's multiple comparison test or student-t-test using GraphPad Prism (GraphPad, CA). Data are means \pm SEM. $p<0.05$ was considered to be statistically significant.

Reporting summary. Further information on research design is available in the Nature Research Reporting Summary linked to this article.

\section{Data availability}

The data that support the findings of this study are available on request from the corresponding author X.L.W. Case Western Reserve University supports the NIH Guidelines for the Sharing of Research Resources including "the Sharing of Biomedical Research Resources: Principles and Guidelines for Recipients of NIH Grants and Contracts". If any intellectual property is pursued, the data will be shared and distributed following advice from the authorities of Case Western Reserve University. The source data underlying Figs. 1, 2g, 2h, 3a-d, 3f, 3g, 4a-f, 5c-f, 5h-k, 5m-p, and Supplementary Figs. 3a-e, 4, 5c-h, 7d, 7e, 7i-k, 8b, 8d, 9a-d, 10b, 10c, 10e, 11b-f, 12a, 12c-e, 13a-d,14, $15 \mathrm{e}-\mathrm{h}, 16,17 \mathrm{~b}-\mathrm{d}, 18 \mathrm{~b}, 18 \mathrm{c}, 19 \mathrm{a}, 19 \mathrm{c}$, and 19e are provided as a Source Data file. Source Data for GWAS in Fig. 1: 10.6084/m9.figshare.11336657 (https://figshare.com/s/ 2d59f2fob7cfd04d31ca). Source Data for GWAS in Supplementary Fig. 2a: 10.6084/m9. figshare.11336666 (https://figshare.com/s/c094ela5c2ea562b6aef). Source Data for GWAS in Supplementary Fig. 2b: 10.6084/m9.figshare.11337272 (https://figshare.com/s/ 1449069e2b80a75b30cf). Source Data for GWAS in Supplementary Fig. 2c: 10.6084/m9. figshare.11337281 (https://figshare.com/s/4cc10442ea2f51333cd7). Source Data for GWAS in Supplementary Fig. 2d: 10.6084/m9.figshare.11337284 (https://figshare.com/s/ 220153dbfce30bca2802). Source Data for GWAS in Supplementary Fig. 2e: 10.6084/m9. figshare.11337290 (https://figshare.com/s/8alf80899f69b462f284)

Received: 3 October 2019; Accepted: 10 December 2019; Published online: 21 January 2020

\section{References}

1. Smith, M. A. Alzheimer disease. Int. Rev. Neurobiol. 42, 1-54 (1998).

2. Tanzi R. E. The genetics of Alzheimer disease. Cold Spring Harb Perspect. Med. 2, a006296 (2012).

3. Cacace, R., Sleegers, K. \& Van Broeckhoven, C. Molecular genetics of earlyonset Alzheimer's disease revisited. Alzheimers Dement 12, 733-748 (2016)

4. Gatz, M. et al. Heritability for Alzheimer's disease: the study of dementia in Swedish twins. J. Gerontol. A Biol. Sci. Med. Sci. 52, M117-M125 (1997).

5. Verheijen J., Sleegers K. Understanding alzheimer disease at the interface between genetics and transcriptomics. Trends Genet.: TIG. 34, 434-447 (2018).

6. Jack, C. R. Jr et al. Tracking pathophysiological processes in Alzheimer's disease: an updated hypothetical model of dynamic biomarkers. Lancet Neurol. 12, 207-216 (2013).

7. Furney, S. J. et al. Genome-wide association with MRI atrophy measures as a quantitative trait locus for Alzheimer's disease. Mol. Psychiatr. 16, 1130-1138 (2011).

8. Biffi, A. et al. Genetic variation and neuroimaging measures in Alzheimer disease. Arch. Neurol. 67, 677-685 (2010).

9. Rajagopalan, P., Hibar, D. P. \& Thompson, P. M. TREM2 and neurodegenerative disease. N. Engl. J. Med 369, 1565-1567 (2013).

10. Giraldo M., et al. Variants in triggering receptor expressed on myeloid cells 2 are associated with both behavioral variant frontotemporal lobar degeneration and Alzheimer's disease. Neurobiol. Aging 34, 2077.e11-8. (2013).

11. Sivakumaran, S. et al. Abundant pleiotropy in human complex diseases and traits. Am. J. Hum. Genet 89, 607-618 (2011).

12. Wagner, G. P. \& Zhang, J. Z. The pleiotropic structure of the genotypephenotype map: the evolvability of complex organisms. Nat. Rev. Genet 12, 204-213 (2011).

13. Zhu, X. et al. Meta-analysis of correlated traits via summary statistics from GWASs with an application in hypertension. Am. J. Hum. Genet. 96, 21-36 (2015).
14. Bulik-Sullivan, B. et al. An atlas of genetic correlations across human diseases and traits. Nat. Genet. 47, 1236-1241 (2015).

15. Harold, D. et al. Genome-wide association study identifies variants at CLU and PICALM associated with Alzheimer's disease. Nat. Genet 41, 1088-1093 (2009).

16. Yashin, A. I. et al. Hidden heterogeneity in Alzheimer's disease: Insights from genetic association studies and other analyses. Exp. Gerontol. 107, 148-160 (2018).

17. Harasty, J. A., Halliday, G. M., Kril, J. J. \& Code, C. Specific temporoparietal gyral atrophy reflects the pattern of language dissolution in Alzheimer's disease. Brain 122(Pt 4), 675-686 (1999).

18. Scahill, R. I., Schott, J. M., Stevens, J. M., Rossor, M. N. \& Fox, N. C. Mapping the evolution of regional atrophy in Alzheimer's disease: unbiased analysis of fluid-registered serial MRI. Proc. Natl Acad. Sci. USA 99, 4703-4707 (2002).

19. Kilimann, I. et al. Subregional basal forebrain atrophy in Alzheimer's disease: a multicenter study. J. Alzheimers Dis. 40, 687-700 (2014).

20. Hibar, D. P. et al. Common genetic variants influence human subcortical brain structures. Nature 520, 224-229 (2015)

21. Lambert, J. C. et al. Meta-analysis of 74,046 individuals identifies 11 new susceptibility loci for Alzheimer's disease. Nat. Genet 45, 1452-1458 (2013).

22. Jagust, W. J. et al. The Alzheimer's Disease neuroimaging initiative positron emission tomography core. Alzheimers Dement 6, 221-229 (2010).

23. Lee, S. et al. Optimal unified approach for rare-variant association testing with application to small-sample case-control whole-exome sequencing studies. Am. J. Hum. Genet 91, 224-237 (2012).

24. $\mathrm{Wu}, \mathrm{M}$. C. et al. Rare-variant association testing for sequencing data with the sequence kernel association test. Am. J. Hum. Genet 89, 82-93 (2011).

25. Lambert, M. P. et al. Monoclonal antibodies that target pathological assemblies of Abeta. J. neurochemistry 100, 23-35 (2007).

26. Oakley, $\mathrm{H}$. et al. Intraneuronal beta-amyloid aggregates, neurodegeneration, and neuron loss in transgenic mice with five familial Alzheimer's disease mutations: potential factors in amyloid plaque formation. J. Neurosci. 26, 10129-10140 (2006)

27. Chishti, M. A. et al. Early-onset amyloid deposition and cognitive deficits in transgenic mice expressing a double mutant form of amyloid precursor protein 695. J. Biol. Chem. 276, 21562-21570 (2001).

28. Radde, R. et al. Abeta42-driven cerebral amyloidosis in transgenic mice reveals early and robust pathology. EMBO Rep. 7, 940-946 (2006).

29. Hsiao, K. et al. Correlative memory deficits, Abeta elevation, and amyloid plaques in transgenic mice. Science 274, 99-102 (1996).

30. Oddo, S. et al. Triple-transgenic model of Alzheimer's disease with plaques and tangles: intracellular Abeta and synaptic dysfunction. Neuron 39, 409-421 (2003).

31. Walsh, D. M., Lomakin, A., Benedek, G. B., Condron, M. M. \& Teplow, D. B. Amyloid beta-protein fibrillogenesis-detection of a protofibrillar intermediate. J. Biol. Chem. 272, 22364-22372 (1997).

32. Akiyama, H. et al. Inflammation and Alzheimer's disease. Neurobiol. Aging 21, 383-421 (2000).

33. O'Leary, T. P., Robertson, A., Chipman, P. H., Rafuse, V. F. \& Brown, R. E. Motor function deficits in the 12 month-old female $5 \times$ FAD mouse model of Alzheimer's disease. Behav. Brain Res 337, 256-263 (2018).

34. Kimura, R. \& Ohno, M. Impairments in remote memory stabilization precede hippocampal synaptic and cognitive failures in 5XFAD Alzheimer mouse model. Neurobiol. Dis. 33, 229-235 (2009).

35. Wang, M. et al. Integrative network analysis of nineteen brain regions identifies molecular signatures and networks underlying selective regional vulnerability to Alzheimer's disease. Genome Med 8, 104 (2016).

36. Bird, A. DNA methylation patterns and epigenetic memory. Genes Dev. 16, 6-21 (2002).

37. Mastroeni, D. et al. Epigenetic changes in Alzheimer's disease: decrements in DNA methylation. Neurobiol. Aging 31, 2025-2037 (2010).

38. Kokjohn, T. A. \& Roher, A. E. Amyloid precursor protein transgenic mouse models and Alzheimer's disease: understanding the paradigms, limitations, and contributions. Alzheimers Dement 5, 340-347 (2009).

39. Kummer, M. P. \& Heneka, M. T. Truncated and modified amyloid-beta species. Alzheimers Res Ther. 6, 28 (2014).

40. Zhao, N., Liu, C. C., Qiao, W. \& Bu, G. Apolipoprotein E, receptors, and modulation of Alzheimer's disease. Biol. Psychiatry 83, 347-357 (2018).

41. Shi, Y. et al. ApoE4 markedly exacerbates tau-mediated neurodegeneration in a mouse model of tauopathy. Nature 549, 523-527 (2017).

42. Liu, E. Y., Li, M., Wang, W. \& Li, Y. MaCH-admix: genotype imputation for admixed populations. Genet Epidemiol. 37, 25-37 (2013).

43. Doshi, J. et al. MUSE: MUlti-atlas region segmentation utilizing ensembles of registration algorithms and parameters, and locally optimal atlas selection. Neuroimage 127, 186-195 (2016).

44. Avants, B. B., Epstein, C. L., Grossman, M. \& Gee, J. C. Symmetric diffeomorphic image registration with cross-correlation: evaluating automated labeling of elderly and neurodegenerative brain. Med. Image Anal. 12, 26-41 (2008). 
45. Ou, Y., Sotiras, A., Paragios, N. \& Davatzikos, C. DRAMMS: deformable registration via attribute matching and mutual-saliency weighting. Med. Image Anal. 15, 622-639 (2011).

46. Purcell, S. Variance components models for gene-environment interaction in twin analysis. Twin Res. 5, 554-571 (2002).

47. Zhang, B. \& Horvath, S. A general framework for weighted gene co-expression network analysis. Stat. Appl. Genet Mol. Biol. 4, Article17 (2005).

48. Barabasi, A. L. \& Albert, R. Emergence of scaling in random networks. Science 286, 509-512 (1999)

49. Willer, C. J., Li, Y. \& Abecasis, G. R. METAL: fast and efficient meta-analysis of genomewide association scans. Bioinformatics 26, 2190-2191 (2010).

50. Nho, K. et al. Comparison of multi-sample variant calling methods for whole genome sequencing. IEEE Int. Conf. Syst. Biol. 2014, 59-62 (2014).

51. Nho, K. et al. Integration of bioinformatics and imaging informatics for identifying rare PSEN1 variants in Alzheimer's disease. BMC Med. Genomics 9 (Suppl. 1), 30 (2016).

52. Ionita-Laza, I., Lee, S., Makarov, V., Buxbaum, J. D. \& Lin, X. Sequence kernel association tests for the combined effect of rare and common variants. Am. J. Hum. Genet. 92, 841-853 (2013).

53. Wang, M. et al. The Mount Sinai cohort of large-scale genomic, transcriptomic and proteomic data in Alzheimer's disease. Sci. Data 5, 180185 (2018).

54. Irizarry, R. A. et al. Exploration, normalization, and summaries of high density oligonucleotide array probe level data. Biostatistics 4, 249-264 (2003).

55. Rostagno, A. \& Ghiso, J. Isolation and biochemical characterization of amyloid plaques and paired helical filaments. Curr. Protoc. Cell Biol. 44, 1-33 (2009).

56. Biancalana, M. \& Koide, S. Molecular mechanism of Thioflavin-T binding to amyloid fibrils. Biochimica Et. Biophysica Acta 1804, 1405-1412 (2010).

57. Xue, C., Lin, T. Y., Chang, D. \& Guo, Z. Thioflavin T as an amyloid dye: fibril quantification, optimal concentration and effect on aggregation. R. Soc. Open Sci. 4, 160696 (2017).

\section{Acknowledgements}

This work is supported by grants from the US National Institutes of Health (RF1AG056320 to X.W.) and the US Alzheimer's Association (AARG-17-499682 to X.W.). Data collection and sharing for this project was funded by the Alzheimer's Disease Neuroimaging Initiative (ADNI) (National Institutes of Health Grant U01 AG024904) and DOD ADNI (Department of Defense award number W81XWH-12-2-0012). ADNI is funded by the National Institute on Aging, the National Institute of Biomedical Imaging and Bioengineering, and through generous contributions from the following: AbbVie, Alzheimer's Association; Alzheimer's Drug Discovery Foundation; Araclon Biotech; BioClinica, Inc.; Biogen; Bristol-Myers Squibb Company; CereSpir, Inc.; Cogstate; Eisai Inc.; Elan Pharmaceuticals, Inc.; Eli Lilly and Company; EuroImmun; F. Hoffmann-La Roche Ltd and its affiliated company Genentech, Inc.; Fujirebio; GE Healthcare; IXICO Ltd.; Janssen Alzheimer Immunotherapy Research \& Development, LLC.; Johnson \& Johnson Pharmaceutical Research \& Development LLC.; Lumosity; Lundbeck; Merck \& Co., Inc.; Meso Scale Diagnostics, LLC.; NeuroRx Research; Neurotrack Technologies; Novartis Pharmaceuticals Corporation; Pfizer Inc.; Piramal Imaging; Servier; Takeda Pharmaceutical Company; and Transition Therapeutics. The
Canadian Institutes of Health Research is providing funds to support ADNI clinical sites in Canada. Private sector contributions are facilitated by the Foundation for the National Institutes of Health (www.fnih.org). The grantee organization is the Northern California Institute for Research and Education, and the study is coordinated by the Alzheimer's Therapeutic Research Institute at the University of Southern California. ADNI data are disseminated by the Laboratory for Neuro Imaging at the University of Southern California.

\section{Author contributions}

X.W. and J.L. conceived and directed the project, interpreted results, and wrote the paper. J.L. and X.Z. contributed to the genetic studies. T.Y. and J.G. contributed to human pathology experiments. T.Y. contributed to the identification of the $A \beta$ binding motif of Aggregatin. J.G. contributed to $A \beta$ in vitro binding and aggregation experiments. T.Y., J.G., and L.W. contributed to all animal related studies. H.F. contributed to electron microscopy study. T.Y., J.L., J.G. L.W., X.Z., and X.W. all contributed to data analysis and paper preparation.

\section{Competing interests}

The authors declare the following competing interests: X.W. has a patent pending regarding the treatment and diagnosis of $\mathrm{AD}$ and related dementia based on Aggregatin.

\section{Additional information}

Supplementary information is available for this paper at https://doi.org/10.1038/s41467019-13962-0.

Correspondence and requests for materials should be addressed to J.L., X.Z. or X.W.

Peer review information Nature Communications thanks Nilufer Ertekin-Taner and the other, anonymous, reviewer(s) for their contribution to the peer review of this work.

Reprints and permission information is available at http://www.nature.com/reprints

Publisher's note Springer Nature remains neutral with regard to jurisdictional claims in published maps and institutional affiliations.

Open Access This article is licensed under a Creative Commons Attribution 4.0 International License, which permits use, sharing, adaptation, distribution and reproduction in any medium or format, as long as you give appropriate credit to the original author(s) and the source, provide a link to the Creative Commons license, and indicate if changes were made. The images or other third party material in this article are included in the article's Creative Commons license, unless indicated otherwise in a credit line to the material. If material is not included in the article's Creative Commons license and your intended use is not permitted by statutory regulation or exceeds the permitted use, you will need to obtain permission directly from the copyright holder. To view a copy of this license, visit http://creativecommons.org/ licenses/by/4.0/.

(C) The Author(s) 2020, corrected publication 2022

\section{The Alzheimer Disease Neuroimaging Initiative}

Michael W. Weiner ${ }^{4}$, Norbert Schuff ${ }^{4}$, Howard J. Rosen ${ }^{4}$, Bruce L. Miller ${ }^{4}$, David Perry ${ }^{4}$, Paul Aisen ${ }^{5}$, Arthur W. Toga ${ }^{5}$, Gustavo Jimenez ${ }^{5}$, Michael Donohue ${ }^{5}$, Devon Gessert ${ }^{5}$, Kelly Harless ${ }^{5}$, Jennifer Salazar ${ }^{5}$, Yuliana Cabrera ${ }^{5}$, Sarah Walter ${ }^{5}$, Lindsey Hergesheimer ${ }^{5}$, Arthur W. Toga ${ }^{5}$, Karen Crawford ${ }^{5}$, Scott Neu ${ }^{5}$, Lon S. Schneider ${ }^{5}$, Sonia Pawluczyk ${ }^{5}$, Mauricio Becerra ${ }^{5}$, Liberty Teodoro ${ }^{5}$, Bryan M. Spann ${ }^{5}$, Paul Aisen ${ }^{5}$, Ronald Petersen ${ }^{6}$, Clifford R. Jack ${ }^{6}$, Matthew Bernstein ${ }^{6}$, Bret Borowski ${ }^{6}$, Jeff Gunter ${ }^{6}$, Matt Senjem ${ }^{6}$, Prashanthi Vemuri ${ }^{6}$, David Jones ${ }^{6}$, Kejal Kantarci ${ }^{6}$, Chad Ward $^{6}$, Sara S. Mason ${ }^{6}$, Colleen S. Albers ${ }^{6}$, David Knopman ${ }^{6}$, Kris Johnson ${ }^{6}$, Neill R. Graff-Radford ${ }^{7}$, Francine Parfitt ${ }^{7}$, Kim Poki-Walker ${ }^{7}$, William Jagust ${ }^{8}$, Susan Landau ${ }^{8}$, John Q. Trojanowki ${ }^{9}$, Leslie M. Shaw ${ }^{9}$, Jason H. Karlawish ${ }^{9}$, David A. Wolk ${ }^{9}$, Sanjeev Vaishnavi ${ }^{9}$, 
Christopher M. Clark ${ }^{9}$, Steven E. Arnold ${ }^{9}$, Virginia Lee ${ }^{9}$, Magdalena Korecka ${ }^{9}$, Michal Figurski ${ }^{9}$, Laurel Beckett ${ }^{10}$, Danielle Harvey ${ }^{10}$, Charles DeCArli ${ }^{10}$, Evan Fletcher ${ }^{10}$, Pauline Maillard ${ }^{10}$, John Olichney ${ }^{10}$, Owen Carmichael $^{10}$, Robert C. Green ${ }^{11}$, Reisa A. Sperling ${ }^{11}$, Keith A. Johnson ${ }^{11}$, Gad A. Marshall11, Andrew J. Saykin ${ }^{12}$, Tatiana M. Foroud ${ }^{12}$, Li Shen ${ }^{12}$, Kelley Faber ${ }^{12}$, Sungeun Kim² ${ }^{12}$ Kwangsik Nho ${ }^{12}$, Martin R. Farlow ${ }^{12}$, Ann Marie Hake ${ }^{12}$, Brandy R. Matthews ${ }^{12}$, Jared R. Brosch ${ }^{12}$, Scott Herring ${ }^{12}$, John Morris ${ }^{13}$, Marc Raichle ${ }^{13}$, David Holtzman ${ }^{13}$, John C. Morris ${ }^{13}$, Nigel J. Cairns ${ }^{13}$, Erin Franklin ${ }^{13}$, Lisa Taylor-Reinwald ${ }^{13}$, Beau Ances ${ }^{13}$, David Winkfield ${ }^{13}$, Maria Carroll ${ }^{13}$, Angela Oliver ${ }^{13}$, Mary L. Creech ${ }^{13}$, Mark A. Mintun ${ }^{13}$, Stacy Schneider ${ }^{13}$, Lew Kuller ${ }^{14}$, Chet Mathis ${ }^{14}$, Oscar L. Lopez ${ }^{14}$, MaryAnn Oakley ${ }^{14}$, Donna M. Simpson ${ }^{14}$, Steven Paul ${ }^{15}$, Norman Relkin ${ }^{15}$, Gloria Chiang ${ }^{15}$, Michael Lin ${ }^{15}$, Lisa Ravdin ${ }^{15}$, Peter Davies ${ }^{16}$, M. Marcel Mesulam ${ }^{17}$, Marek-Marsel Mesulam ${ }^{17}$, Emily Rogalski ${ }^{17}$, Kristine Lipowski ${ }^{17}$, Sandra Weintraub ${ }^{17}$, Borna Bonakdarpour ${ }^{17}$, Diana Kerwin ${ }^{17}$, Chuang-Kuo Wu ${ }^{17}$, Nancy Johnson ${ }^{17}$, Peter J. Snyder ${ }^{18}$, Tom Montine ${ }^{19}$, Michael Donohue ${ }^{20}$, Lean Thal ${ }^{20}$, James Brewer ${ }^{20}$, Helen Vanderswag ${ }^{20}$, Adam Fleisher ${ }^{20}$, Paul Thompson ${ }^{21}$, Ellen Woo ${ }^{21}$, Daniel H.S. Silverman 21, Edmond Teng ${ }^{21}$, Sarah Kremen21, Liana Apostolova21, Kathleen Tingus ${ }^{21}$, Po H. Lu ${ }^{21}$, George Bartzokis ${ }^{21}$, Robert A. Koeppe ${ }^{22}$, Jaimie Ziolkowski ${ }^{22}$, Judith L. Heidebrink ${ }^{22}$, Joanne L. Lord ${ }^{22}$, Norm Foster ${ }^{23}$, Marilyn Albert ${ }^{24}$, Chiadi Onyike 24 , Daniel D'Agostino ${ }^{24}$, Stephanie Kielb24, Joseph Quinn ${ }^{25}$, Lisa C. Silbert ${ }^{25}$, Betty Lind ${ }^{25}$, Jeffrey A. Kaye ${ }^{25}$, Raina Carter ${ }^{25}$, Sara Dolen ${ }^{25}$, Javier Villanueva-Meyer ${ }^{26}$, Valory Pavlik ${ }^{26}$, Nathaniel Pacini26, Ashley Lamb26, Joseph S. Kass ${ }^{26}$, Rachelle S. Doody ${ }^{26}$, Victoria Shibley ${ }^{26}$, Munir Chowdhury ${ }^{26}$, Susan Rountree ${ }^{26}$, Mimi Dang ${ }^{26}$, Yaakov Stern ${ }^{27}$, Lawrence S. Honig ${ }^{27}$, Karen L. Bell ${ }^{27}$, Randy Yeh ${ }^{27}$, Daniel Marson ${ }^{28}$, David Geldmacher ${ }^{28}$, Marissa Natelson ${ }^{28}$, Randall Griffith28, David Clark ${ }^{28}$, John Brockington ${ }^{28}$, Hillel Grossman ${ }^{29}$, Effie Mitsis ${ }^{29}$, Raj C. Shah ${ }^{30}$, Melissa Lamar ${ }^{30}$, Patricia Samuels ${ }^{30}$, Martin Sadowski ${ }^{31}$, Mohammed O. Sheikh ${ }^{31}$, Jamika Singleton-Garvin ${ }^{31}$, Anaztasia Ulysse ${ }^{31}$, Mrunalini Gaikwad ${ }^{31}$, P. Murali Doraiswamy ${ }^{32}$, Olga James ${ }^{32}$, Salvador Borges-Neto ${ }^{32}$, Terence Z. Wong ${ }^{32}$, Edward Coleman ${ }^{32}$, Charles D. Smith ${ }^{33}$, Greg Jicha ${ }^{33}$, Peter Hardy ${ }^{33}$, Riham El Khouli33, Elizabeth Oates ${ }^{33}$, Gary Conrad ${ }^{33}$, Anton P. Porsteinsson ${ }^{34}$, Kim Martin ${ }^{34}$, Nancy Kowalksi ${ }^{34}$, Melanie Keltz ${ }^{34}$,

Bonnie S. Goldstein ${ }^{34}$, Kelly M. Makino ${ }^{34}$, M. Saleem Ismail ${ }^{34}$, Connie Brand ${ }^{34}$, Gaby Thai ${ }^{35}$, Aimee Pierce ${ }^{35}$, Beatriz Yanez ${ }^{35}$, Elizabeth Sosa ${ }^{35}$, Megan Witbracht ${ }^{35}$, Steven Potkin ${ }^{35}$, Kyle Womack ${ }^{36}$, Dana Mathews ${ }^{36}$, Mary Quiceno ${ }^{36}$, Allan I. Levey ${ }^{37}$, James J. Lah ${ }^{37}$, Janet S. Cellar ${ }^{37}$, Jeffrey M. Burns ${ }^{38}$, Russell H. Swerdlow ${ }^{38}$, William M. Brooks ${ }^{38}$, Christopher H. van Dyck ${ }^{39}$, Richard E. Carson ${ }^{39}$, Pradeep Varma ${ }^{39}$, Howard Chertkow ${ }^{40}$, Howard Bergman ${ }^{40}$, Chris Hosein 40 , Raymond Scott Turner ${ }^{41}$, Kathleen Johnson ${ }^{41}$, Brigid Reynolds ${ }^{41}$, Neil Kowall ${ }^{42}$, Ronald Killiany ${ }^{42}$, Andrew E. Budson ${ }^{42}$, Alexander Norbash ${ }^{42}$, Patricia Lynn Johnson ${ }^{42}$, Thomas O. Obisesan ${ }^{43}$, Ntekim E. Oyonumo ${ }^{43}$, Joanne Allard ${ }^{43}$, Olu Ogunlana ${ }^{43}$, Alan Lerner ${ }^{44}$, Paula Ogrocki ${ }^{44}$, Curtis Tatsuoka 44 , Parianne Fatica ${ }^{44}$, Sterling Johnson ${ }^{45}$, Sanjay Asthana 45 , Cynthia M. Carlsson ${ }^{45}$, Jerome Yesavage ${ }^{46}$, Joy L. Taylor ${ }^{46}$, Steven Chao 46 , Barton Lane ${ }^{46}$, Allyson Rosen ${ }^{46}$, Jared Tinklenberg 46 , Douglas W. Scharre ${ }^{47}$, Maria Kataki ${ }^{47}$, Rawan Tarawneh ${ }^{47}$, Earl A. Zimmerman ${ }^{48}$, Dzintra Celmins ${ }^{48}$, David Hart ${ }^{48}$, Laura A. Flashman ${ }^{49}$, Marc Seltzer ${ }^{49}$, Mary L. Hynes ${ }^{49}$, Robert B. Santulli ${ }^{49}$, Kaycee M. Sink ${ }^{50}$, Mia Yang ${ }^{50}$, Akiva Mintz ${ }^{50}$, Delwyn D. Miller ${ }^{51}$, Karen Ekstam Smith ${ }^{51}$, Hristina Koleva ${ }^{51}$, Ki Won Nam 51 , Hyungsub Shim ${ }^{51}$, Susan K. Schultz ${ }^{51}$, Amanda Smith ${ }^{52}$, Christi Leach ${ }^{52}$, Balebail Ashok Raj ${ }^{52}$, Kristin Fargher ${ }^{52}$, Eric M. Reiman ${ }^{53}$, Kewei Chen ${ }^{53}$, Pierre Tariot ${ }^{53}$, Anna Burke ${ }^{53}$, Joel Hetelle ${ }^{53}$, Kathryn DeMarco ${ }^{53}$, Nadira Trncic ${ }^{53}$, Adam Fleisher ${ }^{53}$, Stephanie Reeder ${ }^{53}$, Edward Zamrini ${ }^{54}$, Christine M. Belden ${ }^{54}$, Sherye A. Sirrel ${ }^{54}$, Ranjan Duara 55 , Maria T. Greig-Custo ${ }^{55}$, Rosemarie Rodriguez ${ }^{55}$, Charles Bernick ${ }^{56}$, Donna Munic ${ }^{56}$, Zaven Khachaturian ${ }^{57}$, Neil Buckholtz ${ }^{58}$, John Hsiao ${ }^{58}$, William Potter ${ }^{59}$, Howard Fillit ${ }^{60}$, Franz Hefti ${ }^{61}$, Carl Sadowsky ${ }^{62}$, Teresa Villena ${ }^{62}$, Ging-Yuek Robin Hsiung ${ }^{63}$, Benita Mudge ${ }^{63}$, Vesna Sossi ${ }^{63}$, Howard Feldman ${ }^{63}$, Michele Assaly ${ }^{63}$, Elizabeth Finger ${ }^{64}$, Stephen Pasternack ${ }^{64}$, 
William Pavlosky ${ }^{64}$, Irina Rachinsky ${ }^{64}$, Dick Drost ${ }^{64}$, Andrew Kertesz ${ }^{64}$, Sandra Black ${ }^{65}$, Bojana Stefanovic ${ }^{65}$, Chrinthaka Heyn ${ }^{65}$, Brian R. Ott66, Geoffrey Tremont ${ }^{66}$, Lori A. Daniello66, Courtney Bodge ${ }^{67}$, Stephen Salloway ${ }^{67}$, Paul Malloy ${ }^{67}$, Stephen Correia ${ }^{67}$, Athena Lee ${ }^{67}$, Godfrey D. Pearlson ${ }^{68}$, Karen Blank ${ }^{68}$, Karen Anderson ${ }^{68}$, Vernice Bates ${ }^{69}$, Horacio Capote ${ }^{69}$, Michelle Rainka ${ }^{69}$, Jacobo Mintzer ${ }^{70}$, Kenneth Spicer ${ }^{70}$, David Bachman ${ }^{70}$, Elizabeth Finger ${ }^{71}$, Stephen Pasternak ${ }^{71}$, Irina Rachinsky ${ }^{71}$, John Rogers ${ }^{71}$, Andrew Kertesz ${ }^{71}$, Dick Drost ${ }^{71}$, Elizabeth Finger ${ }^{71}$, Stephen Pasternak ${ }^{71}$, Irina Rachinsky ${ }^{71}$, John Rogers ${ }^{71}$, Andrew Kertesz ${ }^{71}$, Dick Drost ${ }^{71}$, Nunzio Pomara ${ }^{72}$, Raymundo Hernando ${ }^{72}$, Antero Sarrael ${ }^{72}$, Smita Kittur ${ }^{73}$, Michael Borrie ${ }^{74}$, T.-Y. Lee ${ }^{74}$, Rob Bartha ${ }^{74}$, Richard Frank ${ }^{75}$, Nick Fox ${ }^{76}$, Veronika Logovinsky ${ }^{77}$, Maria Corrillo ${ }^{78}$ \& Greg Sorensen 79

\footnotetext{
${ }^{4}$ University of California, San Francisco, CA, USA. ${ }^{5}$ University of Southern California, San Francisco, CA, USA. ${ }^{6}$ Mayo Clinic, Rochester, MN, USA. ${ }^{7}$ Mayo Clinic, Jacksonville, FL, USA. ${ }^{8}$ University of California, Berkeley, CA, USA. ${ }^{9}$ University of Pennsylvania, Philadelphia, PA, USA. ${ }^{10}$ University of California, Davis, CA, USA. ${ }^{11}$ Brigham and Women's Hospital, Boston, MA, USA. ${ }^{12}$ Indiana University, Indianapolis, IN, USA. ${ }^{13}$ Washington University, St. Louis, MO, USA. ${ }^{14}$ University of Pittsburgh, Pittsburgh, PA, USA. ${ }^{15}$ Cornell University, New York, NY, USA. ${ }^{16}$ Albert Einstein College of Medicine of Yeshiva University, New York, NY, USA. ${ }^{17}$ Northwestern University, Chicago, IL, USA. ${ }^{18}$ Brown University, Providence, RI, USA. ${ }^{19}$ University of Washington, Seattle, WA, USA. ${ }^{20}$ University of California, San Diego, CA, USA. ${ }^{21}$ University of California, Los Angeles, CA, USA. ${ }^{22}$ University of Michigan, Ann Arbor, MI, USA. ${ }^{23}$ University of Utah, Salt Lake City, UT, USA. ${ }^{24}$ Johns Hopkins University, Baltimore, MD, USA. ${ }^{25}$ Oregon Health \& Science University, Portland, OR, USA. ${ }^{26}$ Baylor College of Medicine, Houston, TX, USA. ${ }^{27}$ Columbia University Medical Center, New York, NY, USA. ${ }^{28}$ University of Alabama, Birmingham, AL, USA. ${ }^{29}$ Mount Sinai School of Medicine, New York, NY, USA. ${ }^{30}$ Rush University Medical Center, Chicago, IL, USA. ${ }^{31}$ New York University, New York, NY, USA. ${ }^{32}$ Duke University Medical Center, Durham, NC, USA. ${ }^{33}$ University of Kentucky, Lexington, KY, USA. ${ }^{34}$ University of Rochester Medical Center, Rochester, NY, USA. ${ }^{35}$ University of California, Irvine, CA, USA. ${ }^{36}$ University of Texas Southwestern Medical School, Dallas, TX, USA. ${ }^{37}$ Emory University, Atlanta, GA, USA. ${ }^{38}$ University of Kansas Medical Center, Kansas City, KS, USA. ${ }^{39}$ Yale University School of Medicine, New Haven, CT, USA. ${ }^{40}$ McGill University Montreal-Jewish General Hospital, Montreal, QC, Canada. ${ }^{41}$ Georgetown University Medical Center, Washington, DC, USA. ${ }^{42}$ Boston University, Boston, MA, USA. ${ }^{43}$ Howard University, Washington, DC, USA. ${ }^{44}$ University Hospitals, Cleveland, OH, USA. ${ }^{45}$ University of Wisconsin, Madison, WI, USA. ${ }^{46}$ Stanford University, Stanford, CA, USA. ${ }^{47}$ Ohio State University, Columbus, OH, USA. ${ }^{48}$ Albany Medical College, Albany, NY, USA. ${ }^{49}$ Dartmouth-Hitchcock Medical Center, Lebanon, NH, USA. ${ }^{50}$ Wake Forest University Health Sciences, Winston-Salem, NC, USA. ${ }^{51}$ University of lowa College of Medicine, lowa City, IA, USA. ${ }^{52}$ University of South Florida, Health Byrd Alzheimer's Institute, Tampa, FL, USA. ${ }^{53}$ Banner Alzheimer's Institute, Phoenix, AZ, USA. ${ }^{54}$ Banner Sun Health Research Institute, Sun City, AZ, USA. ${ }^{55}$ Wien Center, Miami Beach, FL, USA. ${ }^{56}$ Cleveland Clinic Lou Ruvo Center for Brain Health, Cleveland, OH, USA. ${ }^{57}$ Prevent Alzheimer's Disease, Rockville, MD 2020, USA. ${ }^{58}$ National Institute on Aging, Baltimore, MD, USA. ${ }^{59}$ National Institute of Mental Health, Rockville, MD, USA. ${ }^{60}$ AD Drug Discovery Foundation, New York, NY, USA. ${ }^{61}$ Acumen Pharmaceuticals, Livermore, CA, USA. ${ }^{62}$ Premiere Research Institute, Palm Beach Neurology, West Palm Beach, FL, USA. ${ }^{63}$ U.B.C. Clinic for AD \& Related Disorders, Vancouver, BC, Canada. ${ }^{64}$ Cognitive Neurology - St. Joseph's, London, ON, Canada. ${ }^{65}$ Sunnybrook Health Sciences, Vancouver, ON, Canada. ${ }^{66}$ Rhode Island Hospital, Providence, RI, USA. ${ }^{67}$ Butler Hospital, Butler, PA, USA. ${ }^{68}$ Hartford Hospital, Olin Neuropsychiatry Research Center, Hartford, CT, USA. ${ }^{69}$ Dent Neurologic Institute, Orchard Park, NY, USA. ${ }^{70}$ Medical University South Carolina, Charleston, SC, USA. ${ }^{71}$ St. Joseph's Health Care, London, ON, Canada. ${ }^{72}$ Nathan Kline Institute, Orangeburg, NY, USA. ${ }^{73}$ Neurological Care of CNY, Liverpool, NY, USA. ${ }^{74}$ Parkwood Institute, London, ON, USA. ${ }^{75}$ Richard Frank Consulting, London, UK. ${ }^{76}$ University of London, London, UK. ${ }^{77}$ Eli Lilly and Company, Indianapolis, IN, USA. ${ }^{78}$ Alzheimer's Association, Chicago, IL, USA. ${ }^{79}$ Siemens, Henkestr, DE, Germany
} 